Draft Version OCtober 19, 2010

Preprint typeset using $\mathrm{L}^{A} \mathrm{~T}_{\mathrm{E}} \mathrm{X}$ style emulateapj v. 11/10/09

\title{
EXPLORING SHORT GAMMA-RAY BURSTS AS GRAVITATIONAL-WAVE STANDARD SIRENS
}

\author{
Samaya Nissanke ${ }^{1,2}$, Daniel E. Holz ${ }^{3}$, Scott A. Hughes ${ }^{2}$, Neal Dalal ${ }^{1}$, Jonathan L. Sievers ${ }^{1}$ \\ Draft version October 19, 2010
}

\begin{abstract}
Recent observations support the hypothesis that a large fraction of "short-hard" gamma-ray bursts (SHBs) are associated with the inspiral and merger of compact binaries. Since gravitational-wave (GW) measurements of well-localized inspiraling binaries can measure absolute source distances, simultaneous observation of a binary's GWs and SHB would allow us to directly and independently determine both the binary's luminosity distance and its redshift. Such a "standard siren" (the GW analog of a standard candle) would provide an excellent probe of the nearby $(z \lesssim 0.3)$ universe's expansion, independent of the cosmological distance ladder, thereby complementing other standard candles. Previous work explored this idea using a simplified formalism to study measurement by advanced GW detector networks, incorporating a high signal-to-noise ratio limit to describe the probability distribution for measured parameters. In this paper we eliminate this simplification, constructing distributions with a Markov-Chain Monte-Carlo technique. We assume that each SHB observation gives source sky position and time of coalescence, and we take non-spinning binary neutron star and black hole-neutron star coalescences as plausible SHB progenitors. We examine how well parameters (particularly distance) can be measured from GW observations of SHBs by a range of ground-based detector networks. We find that earlier estimates overstate how well distances can be measured, even at fairly large signal-to-noise ratio. The fundamental limitation to determining distance proves to be a degeneracy between distance and source inclination. Overcoming this limitation requires that we either break this degeneracy, or measure enough sources to broadly sample the inclination distribution. Subject headings: cosmology: distance scale - cosmology: theory - gamma rays: bursts - gravitational waves
\end{abstract}

\section{INTRODUCTION}

\subsection{Overview}

Two multi-kilometer interferometric gravitationalwave (GW) detectors are presently in operation: LIGO4 and Virgd5. They are sensitive to GWs produced by the coalescence of two neutron stars to a distance of roughly $30 \mathrm{Mpc}$, and to the coalescence of a neutron star with a $10 M_{\odot}$ black hole to roughly $60 \mathrm{Mpc}$. Over the next several years, these detectors will undergo upgrades which are expected to extend their range by a factor $\sim 10$. Most estimates suggest that detectors at advanced sensitivity should measure at least a few, and possibly a few dozen, binary coalescences every year (e.g., Kopparapu et al. 2008; Abadie et al. 2010).

It has long been argued that neutron star-neutron star (NS-NS) and neutron star-black hole (NS-BH) mergers are likely to be accompanied by a gamma-ray burst (Eichler et al. 1989). Recent evidence supports the hypothesis that many short-hard gamma-ray bursts (SHBs) are indeed associated with such mergers (Fox et al. 2005, Nakar et al. 2006, Berger et al. 2007, Perley et al. 2008). This suggests that it may be possible to simultaneously measure a binary coalescence in gamma rays (and associated afterglow emission) and in GWs (Dietz 2009).

\footnotetext{
${ }^{1}$ CITA, University of Toronto, 60 St. George St., Toronto, ON, M5S 3H8, Canada

2 Department of Physics and MIT Kavli Institute, 77 Massachusetts Avenue, Cambridge, MA 02139

${ }^{3}$ Theoretical Division, Los Alamos National Laboratory, Los Alamos, NM 87545

${ }^{4}$ http://www.ligo.caltech.edu

${ }^{5}$ http://www.virgo.infn.it
}

The combined electromagnetic and gravitational view of these objects will teach us substantially more than what we learn from either data channel alone. Because GWs track a system's global mass and energy dynamics, it has long been known that measuring GWs from a coalescing binary allows us to determine, in the ideal case, "intrinsic" binary properties such as the masses and spins of its members with exquisite accuracy (Finn \& Chernoff 1993, Cutler \& Flanagan 1994). As we describe in the following subsection, it has also long been appreciated that GWs can determine a system's "extrinsic" properties (Schutz 1986) such as location on the sky and distance to the source. In particular, the amplitude of a binary's GWs directly encodes its luminosity distance. Direct measurement of a coalescing binary could thus be used as a cosmic distance measure: Binary inspiral would be a "standard siren" (the GW equivalent of a standard candle, so-called due to the sound-like nature of GWs) whose calibration depends only on the validity of general relativity (Holz \& Hughes 2005; Dalal et al. 2006).

Unfortunately, GWs alone do not measure extrinsic parameters as accurately as the intrinsic ones. As we describe in more detail in the following section, GW observation of a binary measures a complicated combination of its distance, its position on the sky, and its orientation, with overall fractional accuracy $\sim 1$ /signal-to-noise. As distance is degenerate with these angles, using GWs to measure absolute distance to a source requires a mechanism to break the degeneracy. Associating the GW coalescence waves with a short-hard gamma-ray burst (SHB) is a near-perfect way to break some of these degeneracies.

In this paper we explore the ability of the near-future advanced LIGO-Virgo detector network to constrain bi- 
nary parameters (especially distance), when used in conjunction with electromagnetic observations of the same event (such as an associated SHB). We also examine how well these measurements can be improved if planned detectors in Western Australia (AIGO6) and in Japan's Kamioka mine (LCGT7) are operational. This paper substantially updates and improves upon earlier work (Dalal et al. 2006, hereafter DHHJ06), using a more sophisticated parameter estimation technique. In the next section we review standard sirens, and in Sec. 1.3 we briefly summarize DHHJ06. The next subsection describes the organization and background relevant for the rest of the paper.

\subsection{Standard sirens}

It has long been recognized that GW inspiral measurements could be used as powerful tools for cosmology. Schutz (1986) first demonstrated this by analyzing how binary coalescences allow a direct measurement of the Hubble constant; Marković (1993) and Finn \& Chernoff (1993) subsequently generalized this approach to include other cosmological parameters. More recently, there has been much interest in the measurements enabled when GWs from a merger are accompanied by a counterpart in the electromagnetic spectrum (Bloom et al. 2009, Phinney 2009, Kulkarni \& Kasliwal 2009). In this paper we focus exclusively on GW observations of binaries that have an independent sky position furnished by electromagnetic observations.

We begin by examining gravitational waves from binary inspiral as measured in a single detector. We only present here the lowest order contribution to the waves; in subsequent calculations our results are taken to higher order (see Sec. 2.1). The leading waveform generated by a source at luminosity distance $D_{L}$, corresponding to redshift $z$, is given by

$$
\begin{gathered}
h_{+}=\frac{2(1+z) \mathcal{M}}{D_{L}}[\pi(1+z) \mathcal{M} f]^{2 / 3} \times \\
\quad\left(1+\cos ^{2} \iota\right) \cos 2 \Phi_{N}(t), \\
h_{\times}=-\frac{4(1+z) \mathcal{M}}{D_{L}}[\pi(1+z) \mathcal{M} f]^{2 / 3} \cos \iota \sin 2 \Phi_{N}(t), \\
\Phi_{N}(t)=\Phi_{c}-\left[\frac{t_{c}-t}{5(1+z) \mathcal{M}}\right]^{5 / 8}, \quad f \equiv \frac{1}{\pi} \frac{d \Phi_{N}}{d t} .
\end{gathered}
$$

Here $\Phi_{N}$ is the lowest-order contribution to the orbital phase, $f$ is the GW frequency, and $\mathcal{M}=$ $m_{1}^{3 / 5} m_{2}^{3 / 5} /\left(m_{1}+m_{2}\right)^{1 / 5}$ is the binary's "chirp mass," which sets the rate at which $f$ changes. We use units with $G=c=1$; handy conversion factors are $M_{\odot} \equiv$ $G M_{\odot} / c^{2}=1.47 \mathrm{~km}$, and $M_{\odot} \equiv G M_{\odot} / c^{3}=4.92 \times$ $10^{-6}$ seconds. The angle $\iota$ describes the inclination of the binary's orbital plane to our line-of-sight: $\cos \iota=\hat{\mathbf{L}} \cdot \hat{\mathbf{n}}$, where $\hat{\mathbf{L}}$ is the unit vector normal to the binary's orbital plane, and $\hat{\mathbf{n}}$ is the unit vector along the line-of-sight to the binary. The parameters $t_{c}$ and $\Phi_{c}$ are the time and orbital phase when $f$ diverges in this model. We expect finite size effects to impact the waveform before this divergence is reached.

\footnotetext{
${ }^{6}$ http://www.gravity.uwa.edu.au

7 http://gw.icrr.u-tokyo.ac.jp:8888/lcgt/
}

A given detector measures a linear combination of the polarizations:

$$
h_{\text {meas }}=F_{+}(\theta, \phi, \psi) h_{+}+F_{\times}(\theta, \phi, \psi) h_{\times},
$$

where $\theta$ and $\phi$ describe the binary's position on the sky, and the "polarization angle" $\psi$ sets the inclination of the components of $\hat{\mathbf{L}}$ orthogonal to $\hat{\mathbf{n}}$. The angles $\iota$ and $\psi$ fully specify the orientation vector $\hat{\mathbf{L}}$. For a particular detector geometry, the antenna functions $F_{+}$and $F_{\times}$ can be found in Thorne (1987). In Sec. 2.2 we give a general form for the gravitational waveform without appealing to a specific detector, following the analysis of Cutler \& Flanagan 1994 (hereafter abbreviated CF94).

Several features of Eqs. (1) and (2) are worth commenting upon. First, note that the phase depends on the redshifted chirp mass. Measuring phase thus determines the combination $(1+z) \mathcal{M}($ Finn \& Chernoff 1993), not $\mathcal{M}$ or $z$ independently. To understand this, note that $\mathcal{M}$ controls how fast the frequency evolves: using Eq. (1), we find $\dot{f} \propto f^{11 / 3} \mathcal{M}^{5 / 3}$. The chirp mass enters the system's dynamics as a timescale $\tau_{c}=G \mathcal{M} / c^{3}$. For a source at cosmological distance, this timescale is redshifted; the chirp mass we infer is likewise redshifted. Redshift and chirp mass are inextricably degenerate. This remains true even when higher order effects (see, e.g., Blanchet 2006) are taken into account: parameters describing a binary impact its dynamics as timescales which undergo cosmological redshift, so we infer redshifted values for those parameters. $G W$ observations on their own cannot directly determine a source's redshift.

Next, note that the amplitude depends on $(1+z) \mathcal{M}$, the angles $(\theta, \phi, \iota, \psi)$, and the luminosity distance $D_{L}$. Measuring the amplitude thus measures a combination of these parameters. By measuring the phase, we measure the redshifted chirp mass sufficiently well that $(1+z) \mathcal{M}$ essentially decouples from the amplitude. More concretely, matched filtering the data with waveform templates should allow us to determine the phase with fractional accuracy $\delta \Phi / \Phi \sim 1 /[$ (signal-to-noise $) \times$ (number of measured cycles)]; $(1+z) \mathcal{M}$ should be measured with similar fractional accuracy. NS-NS binaries will radiate roughly $10^{4}$ cycles in the band of advanced LIGO, and NS-BH binaries roughly $10^{3}$ cycles, so the accuracy with which phase and redshifted chirp mass can be determined should be exquisite (Finn \& Chernoff 1993, CF94).

Although $(1+z) \mathcal{M}$ decouples from the amplitude, the distance, position, and orientation angles remain highly coupled. To determine source distance we must break the degeneracy that the amplitude's functional form sets on these parameters. One way to break these degeneracies is to measure the waves with multiple detectors. Studies (Svlvestre 2004; Cavalier et al. 2006; Blair et al. 2008; Fairhurst 2009; Wen \& Chen 2010) have shown that doing so allows us to determine source position to within a few degrees in the best cases, giving some information about the source's distance and inclination.

Perhaps the best way to break some of these degeneracies is to measure the event electromagnetically. An EM signature will pin down the event's position far more accurately than GWs alone. The position angles then decouple, much as the redshifted chirp mass decoupled. Using multiple detectors, we can then determine the 
source's orientation and its distance. This gives us a direct, calibration-free measure of the distance to a cosmic event. The EM signature may also provide us with the event's redshift, directly putting a point on the Hubble diagram. In addition, if modeling or observation give us evidence for beaming of the SHB emission, this could strongly constrain the source inclination.

\subsection{This work and previous analysis}

Our goal is to assess how well we can determine the luminosity distance $D_{L}$ to SHBs under the assumption that they are associated with inspiral GWs. We consider both NS-NS and NS-BH mergers as generators of SHBs, and consider several plausible advanced detector networks: the current LIGO/Virgo network, upgraded to advanced sensitivity; LIGO/Virgo plus the proposed Australian AIGO; LIGO/Virgo plus the proposed Japanese LCGT; and LIGO/Virgo plus AIGO plus LCGT.

The engine of our analysis is a probability function that describes how inferred source parameters $\boldsymbol{\theta}$ should be distributed following GW measurement. (Components $\theta^{a}$ of the vector $\boldsymbol{\theta}$ are physical parameters such as a binary's masses, distance, sky position angles, etc.; our particular focus is on $D_{L}$.) Consider one detector which measures a datastream $s(t)$, containing noise $n(t)$ and a GW signal $h(t, \hat{\boldsymbol{\theta}})$, where $\hat{\boldsymbol{\theta}}$ describes the source's "true" parameters. In the language of Finn (1992), we assume "detection" has already occurred; our goal in this paper is to focus on the complementary problem of "measurement."

As shown by Finn (1992), given a model for our signal $h(t, \boldsymbol{\theta})$, and assuming that the noise statistics are Gaussian, the probability that the parameters $\boldsymbol{\theta}$ describe the data $s$ is

$$
p(\boldsymbol{\theta} \mid s)=p_{0}(\boldsymbol{\theta}) \exp [-((h(\boldsymbol{\theta})-s) \mid(h(\boldsymbol{\theta})-s)) / 2] .
$$

The inner product $(a \mid b)$ describes the noise weighted cross-correlation of $a(t)$ with $b(t)$, and is defined precisely below. The distribution $p_{0}(\boldsymbol{\theta})$ is a prior probability distribution; it encapsulates what we know about our signal prior to measurement. We define $\tilde{\boldsymbol{\theta}}$ to be the parameters that maximize Eq. (3).

DHHJ06 did a first pass on the analysis we describe here. They expanded the exponential to second order in the variables $(\boldsymbol{\theta}-\hat{\boldsymbol{\theta}})$; we will henceforth refer to this as the "Gaussian" approximation (cf. Finn 1992):

$$
\begin{aligned}
& \exp [-(h(\boldsymbol{\theta})-s \mid h(\boldsymbol{\theta})-s) / 2] \simeq \\
& \exp \left[-\frac{1}{2}\left(\frac{\partial h}{\partial \theta^{a}} \mid \frac{\partial h}{\partial \theta^{b}}\right) \delta \theta^{a} \delta \theta^{b}\right],
\end{aligned}
$$

where $\delta \theta^{a}=\theta^{a}-\hat{\theta}^{a}$. In this limit, $\tilde{\boldsymbol{\theta}}=\hat{\boldsymbol{\theta}}$ (at least for uniform priors). The matrix

$$
\Gamma_{a b} \equiv\left(\frac{\partial h}{\partial \theta^{a}} \mid \frac{\partial h}{\partial \theta^{b}}\right)
$$

is the Fisher information matrix. Its inverse $\Sigma^{a b}$ is the covariance matrix. Diagonal entries $\Sigma^{a a}$ are the variance of parameter $\theta^{a}$; off-diagonal entries describe correlations.

The Gaussian approximation to Eq. (3) is known to be accurate when the signal-to-noise ratio (SNR) is large. However, it is not clear what "large" really means (Vallisneri 2008). Given current binary coalescence rate estimates, it is expected that most events will come from $D_{L} \sim$ a few $\times 100 \mathrm{Mpc}$. In such cases, we can expect an advanced detector $\mathrm{SNR} \sim 10$. It is likely that this value is not high enough for the "large SNR" approximation to be appropriate.

In this analysis we avoid the Gaussian approximation. We instead use Markov-Chain Monte-Carlo (MCMC) techniques (in particular, the Metropolis-Hastings algorithm) to explore our parameter distributions. A brief description of this technique is given in Sec. 3, and described in detail in Lewis \& Bridle (2002). We find that the Gaussian approximation to Eq. (3) is indeed failing in its estimate of extrinsic parameters (though it appears to do well for intrinsic parameters such as mass).

\subsection{Organization of this paper}

We begin in Sec. 2 by summarizing how GWs encode the distance to a coalescing binary. We first describe the post-Newtonian ( $\mathrm{PN}$ ) gravitational waveform we use in Sec. 2.1, and then describe how that wave interacts with a network of detectors in Sec. 2.2. Our discussion of the network-wave interaction is heavily based on the notation and formalism used in Sec. 4 of CF94, as well as the analysis of Anderson et al. (2001). Section 2.2 is sufficiently dense that we summarize its major points in Sec. 2.3 before concluding, in Sec. 2.4, with a description of the GW detectors which we include in our analysis.

We outline parameter estimation in Sec. 3. In Sec. 3.1 we describe in more detail how to construct the probability distributions describing parameter measurement. We then give, in Sec. [3.2, a brief description of our selection procedure based on SNR detection thresholds. This procedure sets physically motivated priors for some of our parameters. The Markov-Chain Monte-Carlo technique we use to explore this function is described in Sec. 3.3. How to appropriately average this distribution to give "noise averaged" results and to compare with previous literature is discussed in Sec. 3.4 .

In Sec. 4 we discuss the validation of our code. We begin by attempting to reproduce some of the key results on distance measurement presented in CF94. Because of the rather different techniques used by Cutler \& Flanagan, we do not expect exact agreement. It is reassuring to find, nonetheless, that we can reconstruct with good accuracy all of the major features of their analysis. We then examine how these results change as we vary the amplitude (moving a fiducial test binary to smaller and larger distances), as we vary the number of detectors in our network, and as we vary the source's inclination.

Our main results are given in Sec. 5. We consider several different plausible detector networks and examine measurement errors for two "fiducial" binary systems, comprising either two neutron stars (NS-NS) with physical masses of $m_{1}=m_{2}=1.4 M_{\odot}$, or a neutron star and black hole (NS-BH) system with physical masses $m_{1}=1.4 M_{\odot}$ and $m_{2}=10 M_{\odot}$. Assuming a constant comoving cosmological density, we distribute potential GW-SHB events on the sky, and select from this distribution using a detection threshold criterion set for the entire GW detector network. We summarize some implications of our results in Sec. 6. A more in-depth discussion of these implications, particularly with regard to 
what they imply for cosmological measurements, will be presented in a companion paper.

Throughout this paper, we use units with $G=c=1$. We define the shorthand $m_{z}=(1+z) m$ for any mass parameter $m$.

\section{MEASURING GRAVITATIONAL WAVES FROM INSPIRALING BINARIES}

In this section we review the GW description we use, the formalism describing how these waves interact with a network of detectors, and the properties of the detectors.

\subsection{GWs from inspiraling binaries}

The inspiral and merger of a compact binary's members can be divided into three consecutive phases. The first and longest is a gradual adiabatic inspiral, when the members slowly spiral together due to the radiative loss of orbital energy and angular momentum. PostNewtonian (PN) techniques (an expansion in gravitational potential $M / r$, or equivalently for bound systems, orbital speed $v^{2}$ ) allow a binary's evolution and its emitted GWs to be modeled analytically to high order; see Blanchet (2006) for a review. When the bodies come close together, the PN expansion is no longer valid, and direct numerical calculation is required. Recent breakthroughs in numerical relativity now make it possible to fully model the strong-field, dynamical merger of two bodies into one; see Pretorius (2005), Shibata \& Uryū (2006), and Etienne et al. (2008) for discussion. If the end state is a single black hole, the final waves from the system should be described by a ringdown as the black hole settles down to the Kerr solution.

In this work we are concerned solely with the inspiral, and will accordingly use the $\mathrm{PN}$ waveform to describe our waves. In particular, we use the so-called "restricted" PN waveform; following CF94, the inspiral waveform may be written schematically

$$
h(t)=\operatorname{Re}\left(\sum_{x, m} h_{m}^{x}(t) e^{i m \Phi_{\mathrm{orb}}(t)}\right) .
$$

Here $x$ indicates $\mathrm{PN}$ order $\left[h^{x}\right.$ is computed to $O\left(v^{2 x}\right)$ in orbital speed], $m$ denotes harmonic order (e.g., $m=2$ is quadrupole), and $\Phi_{\text {orb }}(t)=\int^{t} \Omega\left(t^{\prime}\right) d t^{\prime}$ is orbital phase [with $\Omega(t)$ the orbital angular frequency]. The "restricted" waveform neglects all PN amplitude terms beyond the leading one, and considers only the dominant $m=2$ contribution to the phase. The phase is computed to high PN order.

Let the unit vector $\hat{\mathbf{n}}$ point to a binary on the sky (so that the waves propagate to us along $-\hat{\mathbf{n}}$ ), and let the unit vector $\hat{\mathbf{L}}$ denote the normal along the binary's orbital angular momentum. The waveform is fully described by the two polarizations:

$$
\begin{aligned}
h_{+}(t) & =\frac{2 \mathcal{M}_{z}}{D_{L}}\left[\pi \mathcal{M}_{z} f(t)\right]^{2 / 3}\left[1+(\hat{\mathbf{L}} \cdot \hat{\mathbf{n}})^{2}\right] \cos [\Phi(t)], \\
& \equiv \frac{4 \mathcal{M}_{z}}{D_{L}}\left[\pi \mathcal{M}_{z} f(t)\right]^{2 / 3} \mathcal{A}_{+}(\hat{\mathbf{n}}, \hat{\mathbf{L}}) \cos [\Phi(t)] ; \\
h_{\times}(t) & =-\frac{4 \mathcal{M}_{z}}{D_{L}}\left[\pi \mathcal{M}_{z} f(t)\right]^{2 / 3}(\hat{\mathbf{L}} \cdot \hat{\mathbf{n}}) \sin [\Phi(t)] \\
& \equiv \frac{4 \mathcal{M}_{z}}{D_{L}}\left[\pi \mathcal{M}_{z} f(t)\right]^{2 / 3} \mathcal{A}_{\times}(\hat{\mathbf{n}}, \hat{\mathbf{L}}) \sin [\Phi(t)]
\end{aligned}
$$

Equations (7) and (8) are nearly identical to those given in Eq. (1); only the phase $\Phi(t)$ is different, as described below. $\mathcal{M}_{z}$ is the binary's redshifted chirp mass, $D_{L}$ is its luminosity distance, and we have written the inclination angle $\cos \iota$ using the vectors $\hat{\mathbf{n}}$ and $\hat{\mathbf{L}}$. The functions $\mathcal{A}_{+, \times}$compactly gather all dependence on sky position and orientation. In Sec. 2.2 we discuss how these polarizations interact with our detectors.

In these forms of $h_{+}$and $h_{\times}$, the phase is computed to 2nd-post-Newtonian (2PN) order (Blanchet et al. 1995):

$$
\begin{aligned}
\Phi(t)=2 \pi & \int f\left(t^{\prime}\right) d t^{\prime}=2 \pi \int \frac{f}{d f / d t} d f \\
\frac{d f}{d t}=\frac{96}{5} & \pi^{8 / 3} \mathcal{M}_{z}^{5 / 3} f^{11 / 3}\left[1-\left(\frac{743}{336}+\frac{11}{4} \eta\right)\left(\pi M_{z} f\right)^{2 / 3}\right. \\
& +4 \pi\left(\pi M_{z} f\right) \\
& \left.+\left(\frac{34103}{18144}+\frac{13661}{2016} \eta+\frac{59}{18} \eta^{2}\right)\left(\pi M_{z} f\right)^{4 / 3}\right]
\end{aligned}
$$

Higher order results for $d f / d t$ are now known (Blanchet et al. 2002b, a, 2004), but 2PN order will be adequate for our purposes. Since distance measurements depend on accurate amplitude determination, we do not need a highly refined model of the wave's phase. The rate of sweep is dominantly determined by the chirp mass, but there is an important correction due to $\eta=\mu / M=$ $m_{1} m_{2} /\left(m_{1}+m_{2}\right)^{2}$, the reduced mass ratio. Note that $\eta$ is not redshifted; both $\mu$ and $M$ (the reduced mass and total mass, respectively) acquire $(1+z)$ corrections, so their ratio is the same at all $z$. Accurate measurement of the frequency sweep can thus determine both $\mathcal{M}_{z}$ and $\eta\left(\right.$ or $\mathcal{M}_{z}$ and $\left.\mu_{z}\right)$.

We will find it useful to work in the frequency domain, using the Fourier transform $\tilde{h}(f)$ rather than $h(t)$ :

$$
\tilde{h}(f) \equiv \int_{-\infty}^{\infty} e^{2 \pi i f t} h(t) d t .
$$

An approximate result for $\tilde{h}(f)$ can be found using stationary phase (Finn \& Chernoff 1993), which describes the Fourier transform when $f$ changes slowly:

$$
\begin{aligned}
\tilde{h}_{+}(f) & =\sqrt{\frac{5}{96}} \frac{\pi^{-2 / 3} \mathcal{M}_{z}^{5 / 6}}{D_{L}} \mathcal{A}_{+} f^{-7 / 6} e^{i \Psi(f)}, \\
\tilde{h}_{\times}(f) & =\sqrt{\frac{5}{96}} \frac{\pi^{-2 / 3} \mathcal{M}_{z}^{5 / 6}}{D_{L}} \mathcal{A}_{\times} f^{-7 / 6} e^{i \Psi(f)-i \pi / 2} .
\end{aligned}
$$

"Slowly" means that $f$ does not change very much over a single wave period $1 / f$, so that $(d f / d t) / f \ll f$. The validity of this approximation for the waveforms we consider, at least until the last moments before merger, has been demonstrated in previous work (Droz et al. 1999). The phase function $\Psi(f)$ in Eqs. (12) and (13) is given by

$$
\begin{aligned}
\Psi(f)= & 2 \pi f t_{c}-\Phi_{c}-\frac{\pi}{4}+\frac{3}{128}(\pi \mathcal{M} f)^{-5 / 3} \times \\
& {\left[1+\frac{20}{9}\left(\frac{743}{336}+\frac{11}{4} \eta\right)\left(\pi M_{z} f\right)^{2 / 3}\right.} \\
& -16 \pi\left(\pi M_{z} f\right)
\end{aligned}
$$




$$
\left.+10\left(\frac{3058673}{1016064}+\frac{5429}{1008} \eta+\frac{617}{144} \eta^{2}\right)\left(\pi M_{z} f\right)^{4 / 3}\right] \text {. }
$$

As in Eq. (11), $t_{c}$ is called the "time of coalescence" and defines the time at which $f$ diverges within the PN framework; $\Phi_{c}$ is similarly the "phase at coalescence." We assume an abrupt and unphysical transition between inspiral and merger at the innermost stable circular orbit (ISCO), $f_{\mathrm{ISCO}}=\left(6 \sqrt{6} \pi M_{z}\right)^{-1}$. For NS-NS, $f_{\text {ISCO occurs }}$ at high frequencies where detectors have poor sensitivity. As such, we are confident that this abrupt transition has little impact on our results. For NS-BH, $f_{\text {ISCO }}$ is likely to be in a band with good sensitivity, and better modeling of this transition will be important.

In this analysis we neglect effects which depend on spin. In general relativity, spin drives precessions which can "color" the waveform in important ways, and which can have important observational effects (see, e.g.. Vecchio 2004, Lang \& Hughes 2006, van der Sluys et al. 2008). These effects are important when the dimensionless spin parameter, $a \equiv c|\mathbf{S}| / G M^{2}$, is fairly large. Neutron stars are unlikely to spin fast enough to drive interesting precession during the time that they are in the band of GW detectors. To show this, write the moment of inertia of a neutron star as

$$
I_{\mathrm{NS}}=\frac{2}{5} \kappa M_{\mathrm{NS}} R_{\mathrm{NS}}^{2}
$$

where $M_{\mathrm{NS}}$ and $R_{\mathrm{NS}}$ are the star's mass and radius, and the parameter $\kappa$ describes the extent to which its mass is centrally condensed (compared to a uniform sphere). Detailed calculations with different equations of state indicate $\kappa \sim 0.7-1$ [cf. Cook et al. (1994), especially the slowly rotating configurations in their Tables 12, 15, 18, and 21]. For a neutron star whose spin period is $P_{\mathrm{NS}}$, the Kerr parameter is given by

$$
\begin{aligned}
a_{\mathrm{NS}} & =\frac{c}{G} \frac{I_{\mathrm{NS}}}{M_{\mathrm{NS}}^{2}} \frac{2 \pi}{P_{\mathrm{NS}}} \\
& \simeq 0.06 \kappa\left(\frac{R_{\mathrm{NS}}}{12 \mathrm{~km}}\right)^{2}\left(\frac{1.4 M_{\odot}}{M_{\mathrm{NS}}}\right)\left(\frac{10 \mathrm{msec}}{P_{\mathrm{NS}}}\right) .
\end{aligned}
$$

As long as the neutron star spin period is longer than $\sim 10 \mathrm{msec}, a_{\mathrm{NS}}$ is small enough that spin effects can be neglected in our analysis. We should include spin in our models of BH-NS binaries; we leave this to a later analysis. Van der Sluys et al. (2008) included black hole spin effects in an analysis which did not assume known source position. They found that spininduced modulations could help GW detectors to localize a source. This and companion works (Raymond et al. 2009, van der Sluys et al. 2009) suggest that, if position is known, spin modulations could improve our ability to measure source inclination and distance.

Our GWs depend on nine parameters: two masses $\mathcal{M}_{z}$ and $\mu_{z}$, two sky position angles (which set $\hat{\mathbf{n}}$ ), two orientation angles (which set $\hat{\mathbf{L}}$ ), time at coalescence $t_{c}$, phase at coalescence $\Phi_{c}$, and luminosity distance $D_{L}$. When sky position is known, the parameter set is reduced to seven: $\left\{\mathcal{M}_{z}, \mu_{z}, D_{L}, t_{c}, \cos \iota, \psi, \Phi_{c}\right\}$.

\subsection{Measurement of $G W s$ by a detector network}

We now examine how the waves described in Sec. 2.1 interact with a network of detectors. We begin by introducing a geometric convention, which follows that introduced in CF94 and in Anderson et al. (2001). A source's sky position is given by a unit vector $\hat{\mathbf{n}}$ (which points from the center of the Earth to the binary), and its orientation is given by a unit vector $\hat{\mathbf{L}}$ (which points along the binary's orbital angular momentum). We construct a pair of axes which describe the binary's orbital plane:

$$
\hat{\mathbf{X}}=\frac{\hat{\mathbf{n}} \times \hat{\mathbf{L}}}{|\hat{\mathbf{n}} \times \hat{\mathbf{L}}|}, \quad \hat{\mathbf{Y}}=-\frac{\hat{\mathbf{n}} \times \hat{\mathbf{X}}}{|\hat{\mathbf{n}} \times \hat{\mathbf{X}}|} .
$$

With these axes, we define the polarization basis tensors

$$
\begin{aligned}
& \mathbf{e}^{+}=\hat{\mathbf{X}} \otimes \hat{\mathbf{X}}-\hat{\mathbf{Y}} \otimes \hat{\mathbf{Y}}, \\
& \mathbf{e}^{\times}=\hat{\mathbf{X}} \otimes \hat{\mathbf{Y}}+\hat{\mathbf{Y}} \otimes \hat{\mathbf{X}} .
\end{aligned}
$$

The transverse-traceless metric perturbation describing our source's GWs is then

$$
h_{i j}=h_{+} e_{i j}^{+}+h_{\times} e_{i j}^{\times} .
$$

We next characterize the GW detectors. Each detector is an $L$-shaped interferometer whose arms define twothirds of an orthonormal triple. Denote by $\hat{\mathbf{x}}_{a}$ and $\hat{\mathbf{y}}_{a}$ the unit vectors along the arms of the $a$-th detector in our network; we call these the $x$ - and $y$-arms. (The vector $\hat{\mathbf{z}}_{a}=\hat{\mathbf{x}}_{a} \times \hat{\mathbf{y}}_{a}$ points radially from the center of the Earth to the detector's vertex.) These vectors define the response tensor for detector $a$ :

$$
D_{a}^{i j}=\frac{1}{2}\left[\left(\hat{\mathbf{x}}_{a}\right)^{i}\left(\hat{\mathbf{x}}_{a}\right)^{j}-\left(\hat{\mathbf{y}}_{a}\right)^{i}\left(\hat{\mathbf{y}}_{a}\right)^{j}\right] .
$$

The response of detector $a$ to a GW is given by

$$
\begin{aligned}
h_{a} & =D_{a}^{i j} h_{i j} \\
& \equiv e^{-2 \pi i\left(\mathbf{n} \cdot \mathbf{r}_{a}\right) f}\left(F_{a,+} h_{+}+F_{a, \times} h_{\times}\right),
\end{aligned}
$$

where $\mathbf{r}_{\mathbf{a}}$ is the position of the detector $a$ and the factor $\left(\mathbf{n} \cdot \mathbf{r}_{a}\right)$ measures the time of flight between it and the coordinate origin. The second form of Eq. (22) shows how the antenna functions introduced in Eq. (2) are built from the wave tensor and the response tensor.

Our discussion has so far been frame-independent, in that we have defined all vectors and tensors without reference to coordinates. We now introduce a coordinate system for our detectors following Anderson et al. (2001) [who in turn use the WGS-84 Earth model (Althouse et al. 1998)]. The Earth is taken to be an oblate ellipsoid with semi-major axis $a=6.378137 \times 10^{6}$ meters, and semi-minor axis $b=6.356752314 \times 10^{6}$ meters. Our coordinates are fixed relative to the center of the Earth. The $x$-axis (which points along $\mathbf{i}$ ) pierces the Earth at latitude $0^{\circ}$ North, longitude $0^{\circ}$ East (normal to the equator at the prime meridian); the $y$-axis (along $\mathbf{j}$ ) pierces the Earth at $0^{\circ}$ North, $90^{\circ}$ East (normal to the equator in the Indian ocean somewhat west of Indonesia); and the $z$-axis (along $\mathbf{k}$ ) pierces the Earth at $90^{\circ}$ North (the North geographic pole).

A GW source at $(\theta, \phi)$ on the celestial sphere has sky position vector $\hat{\mathbf{n}}$ :

$$
\hat{\mathbf{n}}=\sin \theta \cos \phi \mathbf{i}+\sin \theta \sin \phi \mathbf{j}+\cos \theta \mathbf{k} .
$$


The polarization angle, $\psi$, is the angle (measured clockwise about $\hat{\mathbf{n}}$ ) from the orbit's line of nodes to the source's $\hat{\mathbf{X}}$-axis. In terms of these angles, the vectors $\hat{\mathbf{X}}$ and $\hat{\mathbf{Y}}$ are given by (Anderson et al. 2001)

$$
\begin{aligned}
\hat{\mathbf{X}}= & (\sin \phi \cos \psi-\sin \psi \cos \phi \cos \theta) \mathbf{i} \\
& -(\cos \phi \cos \psi+\sin \psi \sin \phi \cos \theta) \mathbf{j}+\sin \psi \sin \theta \mathbf{k} \\
\hat{\mathbf{Y}}= & (-\sin \phi \sin \psi-\cos \psi \cos \phi \cos \theta) \mathbf{i} \\
& +(\cos \phi \sin \psi-\cos \psi \sin \phi \cos \theta) \mathbf{j}+\cos \psi \sin \theta \mathbf{k}
\end{aligned}
$$

The angle $\phi$ is related to right ascension $\alpha$ by $\alpha=\phi+$ GMST (where GMST is the Greenwich mean sidereal time at which the signal arrives), and $\theta$ is related to declination $\delta$ by $\delta=\pi / 2-\theta$ (cf. Anderson et al. 2001, Appendix B). Combining Eqs. (24) and (25) with Eqs. (18) - (20) allows us to write $h_{i j}$ for a source in coordinates adapted to this problem.

We now similarly describe our detectors using convenient coordinates. Detector $a$ is at East longitude $\lambda_{a}$ and North latitude $\varphi_{a}$ (not to be confused with sky position angle $\phi)$. The unit vectors pointing East, North, and Up for this detector are

$$
\begin{aligned}
& \mathbf{e}_{a}^{\mathrm{E}}=-\sin \lambda_{a} \mathbf{i}+\cos \lambda_{a} \mathbf{j}, \\
& \mathbf{e}_{a}^{\mathrm{N}}=-\sin \varphi_{a} \cos \lambda_{a} \mathbf{i}-\sin \varphi_{a} \sin \lambda_{a} \mathbf{j}+\cos \varphi_{a} \mathbf{k}, \\
& \mathbf{e}_{a}^{\mathrm{U}}=\cos \varphi_{a} \cos \lambda_{a} \mathbf{i}+\cos \varphi_{a} \sin \lambda_{a} \mathbf{j}-\cos \varphi_{a} \mathbf{k} .
\end{aligned}
$$

The $x$-arm of detector $a$ is oriented at angle $\Upsilon_{a}$ North of East, while its $y$-arm is at angle $\Upsilon_{a}+\pi / 2$. Thanks to the Earth's oblateness, the $x$ - and $y$-arms are tilted at angles $\omega_{a}^{x, y}$ to the vertical. The unit vectors $\hat{\mathbf{x}}_{a}, \hat{\mathbf{y}}_{a}$ can thus be written

$$
\begin{aligned}
& \hat{\mathbf{x}}_{a}=\cos \omega_{a}^{x} \cos \Upsilon_{a} \mathbf{e}_{a}^{\mathrm{E}}+\cos \omega_{a}^{x} \sin \Upsilon_{a} \mathbf{e}_{a}^{\mathrm{N}}+\sin \omega_{a}^{x} \mathbf{e}^{\mathrm{U}} \\
& \hat{\mathbf{y}}_{a}=-\cos \omega_{a}^{y} \sin \Upsilon_{a} \mathbf{e}_{a}^{\mathrm{E}}+\cos \omega_{a}^{y} \cos \Upsilon_{a} \mathbf{e}_{a}^{\mathrm{N}}+\sin \omega_{a}^{y} \mathbf{e}^{\mathrm{U}} .
\end{aligned}
$$

Combining Eqs. (29) and (30) with Eq. (21) allows us to write the response tensor for each detector in our network.

\subsection{Summary of the preceding section}

Section 2.2 is sufficiently dense that a brief summary may clarify its key features, particularly with respect to the quantities we hope to measure. From Eq. (22), we find that each detector in our network measures a weighted sum of the two GW polarizations $h_{+}$and $h_{\times}$. Following Cutler (1998), we can rewrite the waveform detector $a$ measures as

$$
h_{a}=\frac{4 \mathcal{M}_{z}}{D_{L}} \mathcal{A}_{p}\left[\pi \mathcal{M}_{z} f(t)\right]^{2 / 3} \cos \left[\Phi(t)+\Phi_{p}\right],
$$

where we have introduced detector $a$ 's "polarization amplitude"

$$
\mathcal{A}_{p}=\sqrt{\left(F_{a,+} \mathcal{A}_{+}\right)^{2}+\left(F_{a, \times} \mathcal{A}_{\times}\right)^{2}},
$$

and its "polarization phase"

$$
\tan \Phi_{p}=\frac{F_{a, \times} \mathcal{A}_{\times}}{F_{a,+} \mathcal{A}_{+}} .
$$

The intrinsic GW phase, $\Phi(t)$, is a strong function of the redshifted chirp mass, $\mathcal{M}_{z}$, the redshifted reduced mass, $\mu_{z}$, the time of coalescence, $t_{c}$, and the phase at coalescence, $\Phi_{c}$. Measuring the phase determines these four quantities, typically with very good accuracy.

Consider for a moment measurements by a single detector. The polarization amplitude and phase depend on the binary's sky position, $(\theta, \phi)$ or $\hat{\mathbf{n}}$, and orientation, $(\psi, \iota)$ or $\hat{\mathbf{L}}$. [They also depend on detector position, $\left(\lambda_{a}, \varphi_{a}\right)$, orientation, $\Upsilon_{a}$, and tilt, $\left(\omega_{a}^{x}, \omega_{a}^{y}\right)$. These angles are known and fixed, so we ignore them in this discussion.] If the angles $(\theta, \phi, \psi, \iota)$ are not known, a single detector cannot separate them, nor can it separate the distance $D_{L}$.

Multiple detectors can, at least in principle, separately determine these parameters. Each detector measures its own amplitude and polarization phase. Combining their outputs, we can fit to the unknown angles and the distance. Various works have analyzed how well this can be done assuming that the position and orientation are completely unknown (Sylvestre 2004; Cavalier et al. 2006; Blair et al. 2008). Van der Sluys et al. (2008) performed such an analysis for measurements of NS-BH binaries, including the effect of orbital precession induced by the black hole. This precession effectively make the angles $\iota$ and $\psi$ time dependent, also breaking the degeneracy among these angles and $D_{L}$.

In what follows, we assume that an electromagnetic identification pins down the angles $(\theta, \phi)$, so that they do not need to be determined from the GW data. We then face the substantially less challenging problem of determining $\psi, \iota$, and $D_{L}$. We will also examine the impact of a constraint on the inclination, $\iota$. Long bursts are believed to be strongly collimated, emitting into jets with opening angles of just a few degrees. Less is known about the collimation of SHBs, but it is plausible that their emission may be primarily along a preferred axis (presumably the progenitor binary's orbital angular momentum axis).

\subsection{GW detectors used in our analysis}

Here we briefly summarize the properties of the GW detectors that we consider.

LIGO: The Laser Interferometer Gravitational-wave Observatory consists of two 4 kilometer interferometers located in Hanford, Washington (US) and Livingston, Louisiana (US). These instruments have achieved their initial sensitivity goals. An upgrade to "advanced" configuration is expected to be completed around 2014, with tuning for best sensitivity to be undertaken in the years following 8 . We show the anticipated noise limits from fundamental noise sources in Fig. 11 for a broad-band tuning (Harry \& LIGO Scientific Collaboration 2010). This spectrum is expected to be dominated by quantum sensing noise above a cut-off at $f<10 \mathrm{~Hz}$, with a contribution from thermal noise in the test mass coatings in the band from 30-200 Hz.

Virgo: The Virgo detector (Acernese \& Virgo Scientific Collaboration 2008) near Pisa, Italy has slightly shorter arms than LIGO (3 kilometers), but should achieve similar advanced sensitivity

${ }^{8}$ http://www.ligo.caltech.edu/advLIGO/scripts/summary.shtml 
TABLE 1

GW DETECTORS (POSITIONS AND ORIENTATIONS).

\begin{tabular}{lccccc}
\hline \hline Detector & East Long. $\lambda$ & North Lat. $\varphi$ & Orientation $\Upsilon$ & $x$-arm tilt $\omega^{x}$ & $y$-arm tilt $\omega^{y}$ \\
\hline LIGO-Han & $-119.4^{\circ}$ & $46.5^{\circ}$ & $126^{\circ}$ & $\left(-6.20 \times 10^{-4}\right)^{\circ}$ & $\left(1.25 \times 10^{-5}\right)^{\circ}$ \\
LIGO-Liv & $-90.8^{\circ}$ & $30.6^{\circ}$ & $198^{\circ}$ & $\left(-3.12 \times 10^{-4}\right)^{\circ}$ & $\left(-6.11 \times 10^{-4}\right)^{\circ}$ \\
Virgo & $10.5^{\circ}$ & $43.6^{\circ}$ & $70^{\circ}$ & $0.0^{\circ}$ & $0.0^{\circ}$ \\
AIGO & $115.7^{\circ}$ & $-31.4^{\circ}$ & $0^{\circ}$ & $0.0^{\circ}$ & $0.0^{\circ}$ \\
LCGT & $137.3^{\circ}$ & $36.4^{\circ}$ & $25^{\circ}$ & $0.0^{\circ}$ \\
& & & & \\
\hline
\end{tabular}

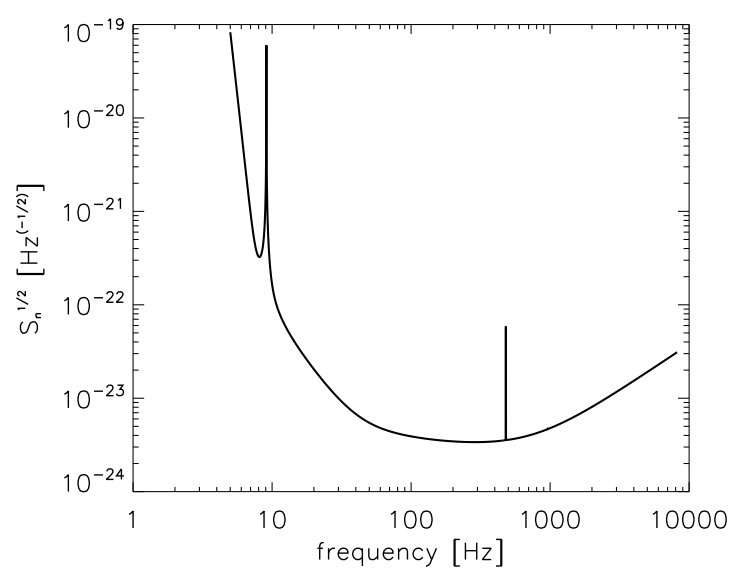

FIG. 1.- Anticipated noise spectrum for Advanced LIGO (Harry \& LIGO Scientific Collaboration 2010; cf. their Fig. 3). Our calculations assume no astrophysically interesting sensitivity below a low frequency cut-off of $10 \mathrm{~Hz}$. The features at $f \simeq 10 \mathrm{~Hz}$ and a few hundred $\mathrm{Hz}$ are resonant modes of the mirror suspensions driven by thermal noise.

on roughly the same timescale as the LIGO detectors? ? For simplicity, we will take Virgo's sensitivity to be the same as LIGO's.

\section{ESTIMATION OF BINARY PARAMETERS}

\subsection{Overview of formalism}

We now give a brief summary of the parameter estimation formalism we use. Further details can be found in Finn (1992), Królak et al. (1993), and CF94.

Assuming detection has occurred, the datastream of detector $a, s_{a}(t)$, has two contributions: The true GW signal $h_{a}(t ; \hat{\boldsymbol{\theta}})$ (constructed by contracting the GW tensor $h_{i j}$ with detector $a$ 's response tensor $D_{a}^{i j}$; cf. Sec. 2.2), and a realization of detector noise $n_{a}(t)$,

$$
s_{a}(t)=h_{a}(t ; \hat{\boldsymbol{\theta}})+n_{a}(t) .
$$

The incident gravitational wave strain depends on (unknown) true parameters $\hat{\boldsymbol{\theta}}$. As in Sec. 1.3, $\hat{\boldsymbol{\theta}}$ is a vector

\footnotetext{
${ }^{9}$ http://www.ego-gw.it/public/virgo/virgo.aspx
}

Our baseline detector network consists of the LIGO Hanford and Livingston sites, and Virgo; these are instruments which are running today, and will be upgraded over the next decade. We also examine the impact of adding two proposed interferometers to this network:

AIGO: The Australian International Gravitational Observatory (Barriga et al. 2010) is a proposed multikilometer interferometer that would be located in Gingin, Western Australia. AIGO's proposed site in Western Australia is particularly favorable due to low seismic and human activity.

LCGT: The Large-scale Cryogenic Gravitational-wave Telescope (Kuroda \& LCGT Collaboration 2010) is a proposed multi-kilometer interferometer that would be located in the Kamioka observatory, 1 kilometer underground. This location takes advantage of the fact that local ground motions tend to decay rapidly as we move away from the Earth's surface. They also plan to use cryogenic cooling to reduce thermal noise.

As with Virgo, we will take the sensitivity of AIGO and LCGT to be the same as LIGO for our analysis. Table 1 gives the location and orientation of these detectors, needed to compute each detector's response function. It's worth mentioning that more advanced detectors are in the early planning stages. Particularly noteworthy is the European proposal for the "Einstein Telescope," currently undergoing design studies. It is being designed to study binary coalescence to high redshift $(z \gtrsim 5)$ (Sathyaprakash et al. 2009).

whose components are binary parameters. Below we use a vector $\mathbf{s}$ whose components $s_{a}$ are the datastreams of each detector. Likewise, $\mathbf{h}$ and $\mathbf{n}$ are vectors whose components are the GW and noise content of each detector.

We assume the noise to be stationary, zero mean, and Gaussian. This lets us categorize it using the spectral density as follows. First, define the noise correlation matrix:

$$
\begin{aligned}
C_{n}(\tau)_{a b} & =\left\langle n_{a}(t+\tau) n_{b}(t)\right\rangle-\left\langle n_{a}(t+\tau)\right\rangle\left\langle n_{b}(t)\right\rangle \\
& =\left\langle n_{a}(t+\tau) n_{b}(t)\right\rangle,
\end{aligned}
$$

where the angle brackets are ensemble averages over noise realizations, and the zero mean assumption gives us the simplified form on the second line. For $a=b$, this is the auto-correlation of detector $a$ 's noise; otherwise, it describes the correlation between detectors $a$ and $b$. The (one-sided) power spectral density matrix is the Fourier 
transform of this:

$$
S_{n}(f)_{a b}=2 \int_{-\infty}^{\infty} d \tau e^{2 \pi i f \tau} C_{n}(\tau)_{a b} .
$$

This is defined for $f>0$ only. For $a=b$, it is the spectral density of noise power in detector $a$; for $a \neq b$, it again describes correlations between detectors. From these definitions, one can show that

$$
\left\langle\tilde{n}_{a}(f) \tilde{n}_{b}\left(f^{\prime}\right)^{*}\right\rangle=\frac{1}{2} \delta\left(f-f^{\prime}\right) S_{n}(f)_{a b}
$$

For Gaussian noise, this statistic completely characterizes our detector noise. No real detector is completely Gaussian, but by using multiple, widely-separated detectors non-Gaussian events can be rejected. For this analysis, we assume the detectors' noises are uncorrelated such that Eq. (37) becomes

$$
\left\langle\tilde{n}_{a}(f) \tilde{n}_{b}\left(f^{\prime}\right)^{*}\right\rangle=\frac{1}{2} \delta_{a b} \delta\left(f-f^{\prime}\right) S_{n}(f)_{a} .
$$

Finally, for simplicity we assume that $S_{n}(f)_{a}$ has the universal shape $S_{n}(f)$ projected for advanced LIGO, shown in Fig. 1].

Many of our assumptions are idealized (Gaussian noise; identical noise spectra; no correlated noise between interferometers), and will certainly not be achieved in practice. These idealizations greatly simplify our analysis, however, and are a useful baseline. It would be useful to revisit these assumptions and understand the quantitative impact that they have on our analysis, but we do not expect a major qualitative change in our conclusions.

The central quantity of interest in parameter estimation is the posterior probability distribution function (PDF) for $\boldsymbol{\theta}$ given detector output $\mathbf{s}$, which is defined as

$$
p(\boldsymbol{\theta} \mid \mathbf{s})=\mathcal{N} p^{(0)}(\boldsymbol{\theta}) \mathcal{L}_{\mathrm{TOT}}(\mathbf{s} \mid \boldsymbol{\theta}) .
$$

$\mathcal{N}$ is a normalization constant, $p^{(0)}(\boldsymbol{\theta})$ is the PDF that represents the prior probability that a measured GW is described by the parameters $\boldsymbol{\theta}$, and $\mathcal{L}_{\mathrm{TOT}}(\mathbf{s} \mid \boldsymbol{\theta})$ is the total likelihood function (e.g., MacKav 2003). The likelihood function measures the relative conditional probability of observing a particular dataset $\mathbf{s}$ given a measured signal $\mathbf{h}$ depending on some unknown set of parameters $\boldsymbol{\theta}$ and given noise $\mathbf{n}$. Because we assume that the noise is independent and uncorrelated at each detector site, we may take the total likelihood function to be the product of the individual likelihoods at each detector:

$$
\mathcal{L}_{\mathrm{TOT}}(\mathbf{s} \mid \boldsymbol{\theta})=\Pi_{a} \mathcal{L}_{a}\left(s_{a} \mid \boldsymbol{\theta}\right),
$$

where $\mathcal{L}_{a}$, the likelihood for detector $a$, is given by (Finn 1992)

$$
\mathcal{L}_{a}(s \mid \boldsymbol{\theta})=e^{-\left(h_{a}(\boldsymbol{\theta})-s_{a} \mid h_{a}(\boldsymbol{\theta})-s_{a}\right) / 2} .
$$

The inner product $(\ldots \mid \ldots)$ on the vector space of signals is defined as

$$
(g \mid h)=2 \int_{0}^{\infty} d f \frac{\tilde{g}^{*}(f) \tilde{h}(f)+\tilde{g}(f) \tilde{h}^{*}(f)}{S_{n}(f)} .
$$

This definition means that the probability of the noise $n(t)$ taking some realization $n_{0}(t)$ is

$$
p\left(n=n_{0}\right) \propto e^{-\left(n_{0} \mid n_{0}\right) / 2} .
$$

For clarity, we distinguish between various definitions of SNR. The true SNR at detector $a$, associated with a given instance of noise for a measurement at a particular detector, is defined as (CF94)

$$
\left(\frac{S}{N}\right)_{a, \text { true }}=\frac{\left(h_{a} \mid s_{a}\right)}{\sqrt{\left(h_{a} \mid h_{a}\right)}} .
$$

This is a random variable with Gaussian PDF of unit variance. For an ensemble of realizations of the detector noise $n_{a}$, the average SNR at detector $a$ is given by

$$
\left(\frac{S}{N}\right)_{a, \text { ave }}=\frac{\left(h_{a} \mid h_{a}\right)}{\operatorname{rms}\left(h_{a} \mid n_{a}\right)}=\left(h_{a} \mid h_{a}\right)^{1 / 2} .
$$

Consequently, we can define the combined true and average SNRs of a coherent network of detectors:

$$
\left(\frac{S}{N}\right)_{\text {true }}=\sqrt{\sum_{a}\left(\frac{S}{N}\right)_{a, \text { true }}^{2}},
$$

and

$$
\left(\frac{S}{N}\right)_{\text {ave }}=\sqrt{\sum_{a}\left(\frac{S}{N}\right)_{a, \text { ave }}^{2}} .
$$

Estimating the parameter set $\boldsymbol{\theta}$ is often done using a "maximum likelihood" method following either a Bayesian (Loredo 1989, Finn 1992, CF94, Poisson \& Will 1995) or frequentist point of view (Królak et al. 1993, CF94). We do not attempt to review these philosophies, and instead refer to Appendix A2 of CF94 for detailed discussion. It is worth noting that, in the GW literature, the "maximum likelihood" or "maximum a posterior" are often interchangeably referred to as "best-fit" parameters. The maximum a posterior is the parameter set $\tilde{\boldsymbol{\theta}}_{\mathrm{MAP}}$ which maximizes the full posterior probability, Eq. (39); likewise, the maximum likelihood is the parameter set $\tilde{\boldsymbol{\theta}}_{\mathrm{ML}}$ which maximizes the likelihood function, Eq. (40).

Following the approach advocated by CF94, we introduce the Bayes estimator $\tilde{\theta}_{\mathrm{BAYES}}^{i}(\mathbf{s})$,

$$
\tilde{\theta}_{\mathrm{BAYES}}^{i}(\mathbf{s}) \equiv \int \theta^{i} p(\boldsymbol{\theta} \mid \mathbf{s}) d \boldsymbol{\theta} .
$$

The integral is performed over the whole parameter set $\boldsymbol{\theta} ; d \boldsymbol{\theta}=d \theta^{1} d \theta^{2} \ldots d \theta^{n}$. Similarly, we define the rms measurement errors $\Sigma_{\text {BAYES }}^{i j}$

$$
\Sigma_{\mathrm{BAYES}}^{i j}=\int\left(\theta^{i}-\tilde{\theta}_{\mathrm{BAYES}}^{i}\right)\left(\theta^{j}-\tilde{\theta}_{\mathrm{BAYES}}^{j}\right) p(\boldsymbol{\theta} \mid \mathbf{s}) d \boldsymbol{\theta}
$$

To understand the meaning of $\tilde{\theta}_{\mathrm{BAYES}}^{i}(\mathbf{s})$, consider a single detector which records an arbitrarily large ensemble of signals. This ensemble will contain a sub-ensemble in which the various $s(t)$ are identical to one another. Each member of the sub-ensemble corresponds to GW signals with different true parameters $\hat{\boldsymbol{\theta}}$, but have noise realizations $n(t)$ that conspire to produce the same $s(t)$. In this case, $\tilde{\theta}_{\mathrm{BAYES}}^{i}(\mathbf{s})$ is the expectation of $\theta^{i}$ averaged over the sub-ensemble. The principle disadvantage of the 
Bayes estimator is the computational cost to evaluate the multi-dimensional integrals in Eqs. (48) and (49).

For large SNR it can be shown that the estimators $\tilde{\boldsymbol{\theta}}_{\mathrm{ML}}$, $\tilde{\boldsymbol{\theta}}_{\mathrm{MAP}}$, and $\tilde{\boldsymbol{\theta}}_{\mathrm{BAYES}}$ agree with one another (CF94), and that Eq. (39) is well-described by a Gaussian form [cf. Eq. (4)]. However, as illustrated in Sec. IVD of CF94, effects due to prior information and which scale nonlinearly with 1 /SNR contribute significantly at low SNR. The Gaussian approximation then tends to underestimate measurement errors by missing tails or multimodal structure in posterior distributions.

We emphasize that in this analysis we do not consider systematic errors that occur due to limitations in our source model or to gravitational lensing effects. A framework for analyzing systematic errors in GW measurements has recently been presented by Cutler \& Vallisneri (2007). An important follow-on to this work will be to estimate systematic effects and determine whether they significantly change our conclusions.

\subsection{Binary Selection and Priors}

We now describe how we generate a sample of detectable GW-SHB events. We assume a constant comoving density (Peebles 1993, Hogg 1999) of GW-SHB events, in a $\Lambda$ CDM Universe with $H_{0}=$ $70.5 \mathrm{~km} / \mathrm{sec} / \mathrm{Mpc}, \Omega_{\Lambda}=0.726$, and $\Omega_{m}=0.2732$ (Komatsu et al. 2009). We distribute $10^{6}$ binaries uniformly in volume with random sky positions and orientations to redshift $z=1\left(D_{L} \simeq 6.6 \mathrm{Gpc}\right)$. We then compute the average SNR, Eq. (45), for each binary at each detector, and use Eq. (47) to compute the average total SNR for each network we consider. We assume prior knowledge of the merger time (since we have assumed that the inspiral is correlated with a SHB), so we set a threshold SNR for the total detector network, $\mathrm{SNR}_{\text {total }}=7.5$ (see discussion in DHHJ06). This is somewhat reduced from the threshold we would set in the absence of a counterpart, since prior knowledge of merger time and source position reduces the number of search templates we need by a factor $\sim 10^{5}$ Kochanek \& Piran 1993, Owen 1996). Using the average SNR to set our threshold introduces a slight error into our analysis, since the true SNR will differ from the average. Some events which we identify as above threshold could be moved below threshold due to a measurement's particular noise realization. However, some sub-threshold events will likewise be moved above threshold, and the net effect is not expected to be significant.

Our threshold selects detectable GW-SHB events for each detector network. We define "total detected binaries" to mean binaries which are detected by a network of all five detectors - both LIGO sites, Virgo, AIGO, and LCGT. Including AIGO and LCGT substantially improves the number detected, as compared to just using the two LIGO detectors and Virgo. Assuming that all binary orientations are equally likely given an SHB (i.e., no beaming), we find that a LIGO-Virgo network detects $50 \%$ of the total detected binaries; LIGO-VirgoAIGO detects $74 \%$ of the total; and LIGO-Virgo-LCGT detects $72 \%$ of the total. Figure 2 shows the sky distribution of detected binaries for various detector combinations. Networks which include LCGT tend to have rather uniform sky coverage. Those with AIGO cover
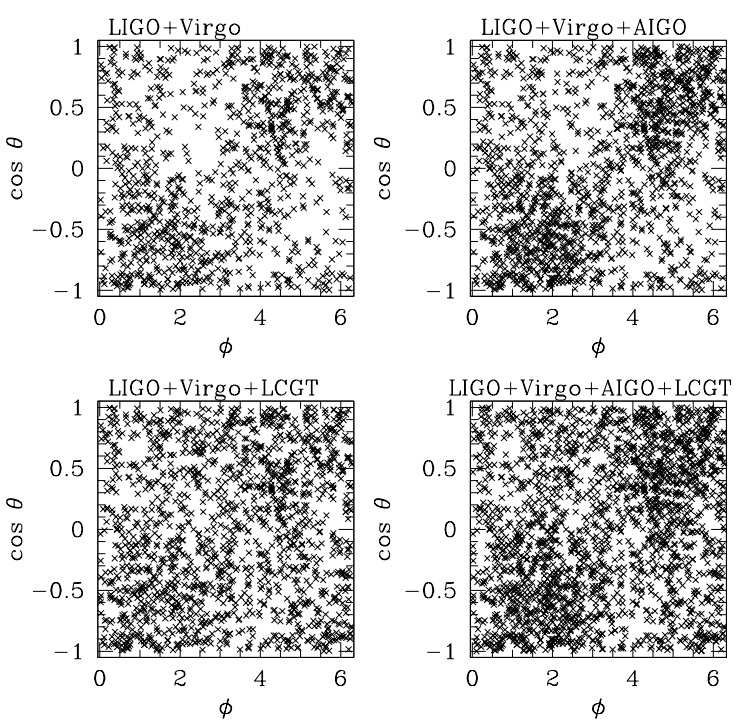

FIG. 2.- Detected NS-NS binaries for our various detector networks as a function of sky position $(\cos \theta, \phi)$. The lower right panel shows the binaries detected by a five-detector network (both LIGO sites, Virgo, AIGO, and LCGT). We find that LIGO plus Virgo (our "base" network) only detects $50 \%$ of the five-detector events; LIGO, Virgo, and AIGO detect $74 \%$ of these events; and LIGO, Virgo, and LCGT, detect $72 \%$ of these events. Detections are more uniformly distributed on the sky in networks that include LCGT; AIGO improves coverage in two of the sky's quadrants. Our coordinate $\phi$ is related to right ascension $\alpha$ by $\phi=\alpha-$ GMST, where GMST is Greenwich Mean Sidereal Time; $\theta$ is related to declination $\delta$ by $\theta=\pi / 2-\delta$.

the quadrants $\cos \theta>0, \phi>\pi$ and $\cos \theta<0, \phi<\pi$ particularly well.

Our selection method implicitly sets a prior distribution on our parameters. For example, the thresholding procedure results in a significant bias in detected events toward face-on binaries, with $\hat{\mathbf{L}} \cdot \hat{\mathbf{n}} \rightarrow \pm 1$. Figure 3 shows the distribution of detectable NS-NS binaries for the parameters $\left(\cos \iota, D_{L}\right)$. Since we use an unrealistic mass distribution $\left(1.4 M_{\odot}-1.4 M_{\odot}\right.$ NS-NS and $1.4 M_{\odot}-10 M_{\odot}$ NS-BH binaries), instead of a more astrophysically realistic distribution, the implicit mass prior is uninteresting. Figure 4 shows the average total SNR versus the true $D_{L}$ of our sample of detectable NS-NS and NS-BH binaries for our "full" network (LIGO, Virgo, AIGO, LCGT). Very few detected binaries have SNR above 30 for NS$\mathrm{NS}$, and above 70 for NS-BH. It is interesting to note the different detectable ranges between the two populations: NS-BH binaries are detectable to over twice the distance of NS-NS binaries.

We are also interested in seeing the impact that prior knowledge of SHB collimation may have on our ability to measure these events. To date there exist only two tentative observations which suggest that SHBs may be collimated (Grupe et al. 2006, Burrows et al. 2006, Soderberg et al. 2006); we therefore present results for moderate collimation and for isotropic SHB emission. To obtain a sample of beamed SHBs, we assume that the burst emission is collimated along the orbital angular momentum axis, where baryon loading is minimized. Following DHHJ06, we use a distribution for $\cos \iota \equiv v$ of $d P / d v \propto \exp \left[-(1-v)^{2} / 2 \sigma_{v}^{2}\right]$, with $\sigma_{v}=0.05$. This corresponds to a beamed population with $68 \%$ of its distri- 


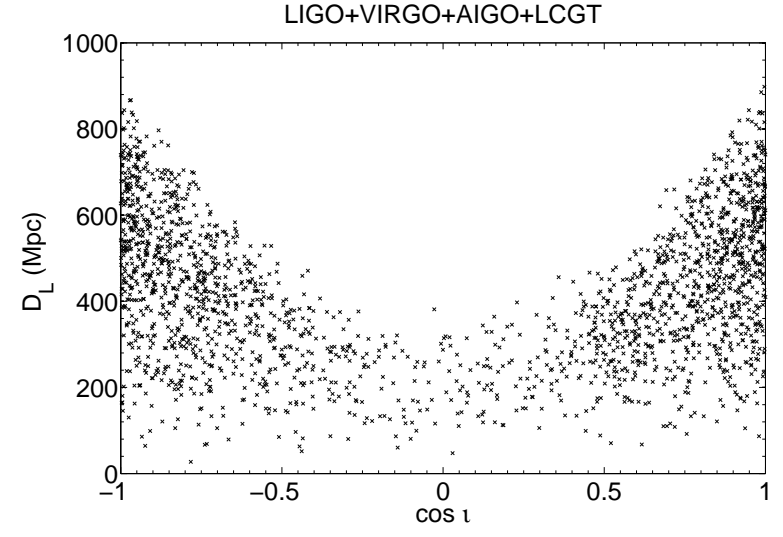

FIG. 3.- The 2-D marginalized prior distribution in luminosity distance $D_{L}$ and cosine inclination $\cos \iota$. Each point represents a detected NS-NS binary for a network comprising all five detectors. Notice the bias toward detecting face-on binaries $(\cos \iota \rightarrow \pm 1)$ they are detected to much larger distances than edge-on $(\cos \iota \rightarrow$ $0)$.
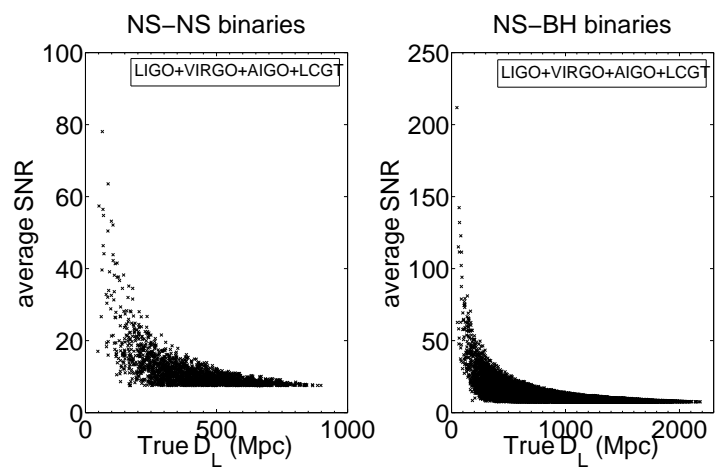

FIG. 4.- Average network SNR versus luminosity distance of the total detected NS-NS and NS-BH binaries. This assumes an idealized network consisting of both LIGO detectors, Virgo, AIGO, and LCGT. Left panel shows all detected NS-NS binaries (one point with SNR above 100 is omitted); right panel shows all detected NS-BH binaries (one point with SNR above 350 is omitted). Notice the different axis scales: NS-BH binaries are detected to more than twice the distance of NS-NS. The threshold SNR for the total detector network is $7.5, \mathrm{SNR}_{\text {total }}=7.5$.

bution having an opening jet angle within roughly $25^{\circ}$. We construct a beamed subsample by selecting events from the total sample of detected events such that the final distribution in inclination angle follows $d P / d v$. Joint measurements of SHBs and GW-driven inspirals should enable us to constrain beaming angles by comparing the measured rates for these two populations.

\subsection{Markov-Chain Monte-Carlo approach}

The principle disadvantage of the Bayes estimators $\tilde{\theta}_{\mathrm{BAYES}}^{i}$ and $\Sigma_{\mathrm{BAYES}}^{i j}$ is the high computational cost of evaluating the multi-dimensional integrals which define them, Eqs. (48) and (49). To get around this problem, we use Markov-Chain MonteCarlo (MCMC) methods to explore the PDFs describing the seven parameters $\left\{\mathcal{M}_{c}, \mu, D_{L}, \cos \iota, \psi, t_{c}, \Phi_{c}\right\}$. MCMC methods are widely used in diverse astrophysical applications, ranging from high precision cosmology (e.g. Dunkley et al. 2009, Sievers et al. 2009) to extra-solar planet studies (e.g. Ford 2005, Winn et al. 2007). They have seen increased use in GW measurement and parameter estimation studies in re- cent years (e.g., Stroeer et al. 2006, Wickham et al. 2006, Cornish \& Porter 2007, Porter \& Cornish 2008, Röver et al. 2007, van der Sluys et al. 2008).

MCMC generates a random sequence of parameter states that sample the posterior distribution, $p(\boldsymbol{\theta} \mid \mathbf{s})$. Let the $n$th sample in the sequence be $\boldsymbol{\theta}^{(n)}$. If one draws a total of $N$ random samples, Eqs. (48) and (49) can then be approximated as sample averages:

$$
\begin{aligned}
\tilde{\theta}_{\mathrm{BAYES}}^{i} & \simeq \frac{1}{N} \sum_{n=1}^{N}\left(\theta^{i}\right)^{(n)} \\
\Sigma_{\mathrm{BAYES}}^{i j} & \simeq \frac{1}{N} \sum_{n=1}^{N}\left(\tilde{\theta}_{\mathrm{BAYES}}^{i}-\left(\theta^{i}\right)^{(n)}\right)\left(\tilde{\theta}_{\mathrm{BAYES}}^{j}-\left(\theta^{j}\right)^{(n)}\right) .
\end{aligned}
$$

The key to making this technique work is drawing a sequence that represents the posterior PDF. We use the Metropolis-Hastings algorithm to do this (Metropolis et al. 1953, Hastings 1970); see Neal (1993), Gilks et al. (1996), MacKav (2003), and Christensen et al. (2004) for in-depth discussion. The MCMC algorithm we use is based on a generic version of CosmoMC10, described in Lewis \& Bridle (2002).

Appropriate priors are crucial to any MCMC analysis. We take the prior distributions in chirp mass $\mathcal{M}_{z}$, reduced mass $\mu_{z}$, polarization angle $\psi$, coalescence time $t_{c}$, and coalescence phase $\Phi_{c}$ to be flat over the region of sample space where the binary is detectable according to our selection procedure. More specifically, we choose

- $p^{(0)}\left(\mathcal{M}_{z}\right)=$ constant over the range $\left[1 M_{\odot}, 2 M_{\odot}\right]$ for NS-NS; and over the range $\left[2.5 M_{\odot}, 4.9 M_{\odot}\right]$ for NS-BH. (The true chirp masses in the binaries' rest frames are $1.2 M_{\odot}$ for NS-NS and $3.0 M_{\odot}$ for NS-BH.)

- $p^{(0)}\left(\mu_{z}\right)=$ constant over the range $\left[0.3 M_{\odot}, 2 M_{\odot}\right]$ for NS-NS; and over the range $\left[0.5 M_{\odot}, 3.5 M_{\odot}\right]$ for NS-BH. (The true reduced masses in the binaries' rest frames are $0.7 M_{\odot}$ for NS-NS and $1.2 M_{\odot}$ for NS-BH.)

- $p^{(0)}(\psi)=$ constant over the range $[0, \pi]$.

- $p^{(0)}\left(t_{c}\right)=$ constant over the range $[-100 \mathrm{sec}, 100 \mathrm{sec}]$. Since we assume that $t_{c}$ is close to the time of the SHB event, it is essentially the time offset between the system's final GWs and its SHB photons. We find that the range in $t_{c}$ we choose is almost irrelevant, as long as the prior is flat and includes the true value. No matter how broad we choose the prior in $t_{c}$, our posterior PDF ends up narrowly peaked around $\hat{t}_{c}$.

- $p^{(0)}\left(\Phi_{c}\right)=$ constant over the range $[0,2 \pi]$.

The prior distribution for $D_{L}$ is inferred by taking the density of SHBs to be uniform per unit comoving volume over the luminosity distance range $[0,2 \mathrm{Gpc}]$ for NS-NS binaries, and over the range $[0,5 \mathrm{Gpc}]$ for NS$\mathrm{BH}$ binaries. For our sample with isotropic inclination

10 See http://cosmologist.info/cosmomc/ 
distribution, we put $p^{(0)}(\cos \iota)=$ constant over the range $[-1,1]$. When we assume SHB collimation, our prior in $\cos \iota \equiv v$ is the same as the one that we used in our selection procedure discussed previously:

$$
\frac{d p^{(0)}}{d v}(v) \propto e^{-(1-v)^{2} / 2 \sigma_{v}^{2}}
$$

with $\sigma_{v}=0.05$.

We then map out full distributions for each of our seven parameters, assessing the mean values [Eq. (48)] and the standard deviations [Eq. (49)]. We generate four chains which run in parallel on the CITA "Sunnyvale" Cluster. Each chain runs for a maximum of $10^{7}$ steps; we find that the mean and median number of steps are $\sim 10^{5}$ and $\sim 10^{4}$, respectively. Each evaluation of the likelihood function takes $\sim 0.3$ seconds. We use the first $30 \%$ of a chain's sample states for "burn in," and discard that data. Our chains start at random offset parameter values, drawn from Gaussians centered on the true parameter values. We assess convergence by testing whether the multiple chains have produced consistent parameter distributions. Following standard practice, we use the Gelman-Rubin convergence criterion, defining a sequence as "converged" if the statistic $R<1.1$ on the last half of our samples; see Gelman \& Rubin (1992) for more details. We use convergence as our stopping criterion. Each simulation for every binary runs for an hour to forty-eight hours; the mean and median runtime are eight and three hours, respectively.

\subsection{The "averaged" posterior PDF}

Central to the procedure outlined above is the use of the datastream $\mathbf{s}=\mathbf{h}(\boldsymbol{\theta})+\mathbf{n}$ which enters the likelihood function $\mathcal{L}_{\mathrm{TOT}}(\mathbf{s} \mid \boldsymbol{\theta})$. The resulting posterior PDF, and the parameters one infers, thus depend on the noise $\mathbf{n}$ which one uses. One may want to evaluate statistics that are in a well-defined sense "typical" given the average noise properties, rather than depending on a particular noise instance. Such averaging is appropriate, for example, when forecasting how well an instrument should be able to measure the properties of a source or process. We have also found it is necessary to average when trying to compare our MCMC code's output with previous work.

As derived below, the averaged posterior PDF takes a remarkably simple form: It is the "usual" posterior PDF, Eq. (39) with the noise $\mathbf{n}$ set to zero. This does not mean that one ignores noise when constructing the averaged PDF; one still relates signal amplitude to typical noise by the average SNR, Eq. (45). As such, the averaged statistics will show an improvement in measurement accuracy as SNR is increased.

To develop a useful notion of averaged posterior PDF, consider the hypothetical (and wholly unrealistic) case in which we measure a signal using $M$ different noise realizations for the same event. The joint likelihood for these measurements is

$$
\mathcal{L}_{\mathrm{TOT}}^{\text {joint }}\left(\mathbf{s}_{1}, \mathbf{s}_{2}, \ldots \mathbf{s}_{M} \mid \boldsymbol{\theta}\right)=\prod_{i=1}^{M} \mathcal{L}_{\mathrm{TOT}}\left(\mathbf{s}_{i} \mid \boldsymbol{\theta}\right) .
$$

Let us define the "average" PDF as the product of the prior distribution of the parameters multiplied by the geometric mean of the likelihoods which describe these measurements:

$$
p_{\text {ave }}(\boldsymbol{\theta} \mid \mathbf{s}) \equiv \mathcal{N} p^{(0)} \mathcal{L}_{\text {TOT }}^{\text {joint }}\left(\mathbf{s}_{1}, \mathbf{s}_{2}, \ldots \mathbf{s}_{M} \mid \boldsymbol{\theta}\right)^{1 / M} .
$$

Expanding this definition, we find

$$
p_{\text {ave }}(\boldsymbol{\theta} \mid \mathbf{s}) \equiv \mathcal{N} p^{(0)} \prod_{i=1}^{M}\left[\mathcal{L}_{\mathrm{TOT}}\left(\mathbf{s}_{i} \mid \boldsymbol{\theta}\right)\right]^{1 / M},
$$

where the subscript $i$ denotes the $i$ th noise realization in our set of $M$ observations. The "ensemble average likelihood function" can in turn be expanded as

$$
\begin{aligned}
\prod_{i=1}^{M}\left[\mathcal{L}_{\mathrm{TOT}}\left(\mathbf{s}_{i} \mid \boldsymbol{\theta}\right)\right]^{1 / M} & =\prod_{a} \prod_{i=1}^{M}\left[\mathcal{L}_{a}\left(s_{a, i} \mid \boldsymbol{\theta}\right)\right]^{1 / M} \\
& =\prod_{a} \prod_{i=1}^{M} e^{-\left(h_{a}(\boldsymbol{\theta})-s_{a, i} \mid h_{a}(\boldsymbol{\theta})-s_{a, i}\right) / 2 M} \\
& =\prod_{a} e^{-\left(h_{a}(\boldsymbol{\theta})-h_{a}(\hat{\boldsymbol{\theta}}) \mid h_{a}(\boldsymbol{\theta})-h_{a}(\hat{\boldsymbol{\theta}})\right) / 2} \\
& \times \prod_{i=1}^{M} \exp \left[\frac{1}{M}\left(n_{a, i} \mid h_{a}(\boldsymbol{\theta})-h_{a}(\hat{\boldsymbol{\theta}})\right)\right] \\
& \times \prod_{i=1}^{M} \exp \left[-\frac{1}{2 M}\left(n_{a, i} \mid n_{a, i}\right)\right] . \quad(56)
\end{aligned}
$$

By taking $M$ to be large, the last two lines of Eq. (56) can be evaluated as follows:

$$
\begin{aligned}
\prod_{i=1}^{M} \exp [ & \left.\frac{1}{M}\left(n_{a, i} \mid h_{a}(\boldsymbol{\theta})-h_{a}(\hat{\boldsymbol{\theta}})\right)\right] \\
& =\exp \left[\frac{1}{M} \sum_{i=1}^{M}\left(n_{a, i} \mid h_{a}(\boldsymbol{\theta})-h_{a}(\hat{\boldsymbol{\theta}})\right)\right] \\
& \simeq \exp \left[\left\langle\left(n_{a} \mid h_{a}(\boldsymbol{\theta})-h_{a}(\hat{\boldsymbol{\theta}})\right)\right\rangle\right] \\
& =1 .
\end{aligned}
$$

Here, $\langle\ldots\rangle$ denotes an ensemble average over noise realizations (cf. Sec. 3.1), and we have used the fact that our noise has zero mean. Similarly, we find

$$
\begin{aligned}
\prod_{i=1}^{M} \exp \left[-\frac{1}{2 M}\left(n_{a, i} \mid n_{a, i}\right)\right] & =\exp \left[-\frac{1}{2 M} \sum_{i=1}^{M}\left(n_{a, i} \mid n_{a, i}\right)\right] \\
& \simeq \exp \left[-\frac{1}{2}\left\langle\left(n_{a} \mid n_{a}\right)\right\rangle\right] \\
& =e^{-1} .
\end{aligned}
$$

This uses $\left\langle\left(n_{a} \mid n_{a}\right)\right\rangle=2$, which can be proved using the noise properties (35), (36), and (37).

Putting all this together, we finally find

$$
p_{\text {ave }}(\boldsymbol{\theta} \mid \mathbf{s})=\mathcal{N} p^{0}(\boldsymbol{\theta}) \prod_{a} e^{-\left(h_{a}(\boldsymbol{\theta})-h_{a}(\hat{\boldsymbol{\theta}}) \mid h_{a}(\boldsymbol{\theta})-h_{a}(\hat{\boldsymbol{\theta}})\right) / 2}
$$

where we have absorbed $e^{-1}$ into the normalization $\mathcal{N}$. The posterior PDF, averaged over noise realizations, is simply obtained by evaluating Eq. (39) with the noise $\mathbf{n}$ set to zero. 


\section{RESULTS I: VALIDATION AND TESTING}

We now validate and test our MCMC code against results from CF94. In particular, we examine the posterior PDF for the NS-NS binary which was studied in detail in CF94. We also explore the dependence of distance measurement accuracies on the detector network and luminosity distance, focusing on the strong degeneracy that exists between $\cos \iota$ and $D_{L}$.

\subsection{Comparison with CF94}

Validation of our MCMC results requires comparing to work which goes beyond the Gaussian approximation and Fisher matrix estimators. In Section IVD of CF94, Cutler \& Flanagan investigate effects that are non-linear in 1/SNR. They show that such effects have a significant impact on distance measurement accuracies for low SNR. In particular, they find that Fisher-based estimates understate distance measurement errors for a network of two LIGO detectors and Virgo.

Because they go beyond a Fisher matrix analysis, the results of CF94 are useful for comparing to our results. Their paper is also useful in that they take source position to be known. Our approach is sufficiently different from CF94 that we do not expect perfect agreement, however. The most important difference is that we directly map out the posterior PDF and compute sample averages using Eqs. (48) and (49), for the full parameter set $\left\{\mathcal{M}_{z}, \mu_{z}, D_{L}, \cos \iota, \psi, t_{c}, \Phi_{c}\right\}$. In contrast, CF94 estimate measurement errors only for $D_{L}$, using an approximation on an analytic Bayesian derivation of the marginalized PDF for $D_{L}$. Specifically, Cutler \& Flanagan expand the exponential factor in Eq. (39) beyond second order in terms of some "best-fit" maximum likelihood parameters. Their approximation treats strong correlations between the parameters $D_{L}$ and $\cos \iota$ that are non-linear in 1/SNR. However, other correlations between $D_{L}$ and $\left(\psi, \phi_{c}\right)$ are only considered to linear order. They obtain an analytic expression for the posterior PDF of the variables $D_{L}$ and $\cos \iota$ in terms of their "best-fit" maximum-likelihood values $\tilde{D}_{L}$ and $\cos \tilde{\iota}$ [see Eq. (4.57) of CF94]. The marginalized 1-D posterior PDFs for $D_{L}$ are then computed by numerically integrating over $\cos \iota$. The 1-D marginalized PDF we compute in parameter $\theta_{i}$ is

$$
p_{\text {marg }}\left(\theta_{i} \mid \mathbf{s}\right)=\int \ldots \int p(\boldsymbol{\theta} \mid \mathbf{s}) d \theta_{1} \ldots d \theta_{i-1} d \theta_{i+1} \ldots d \theta_{N}
$$

where $p(\boldsymbol{\theta} \mid \mathbf{s})$ is the posterior PDF given by Eq. (39) and $N$ is the number of dimensions of our parameter set.

In addition to this rather significant difference in techniques, there are some minor differences which also affect our comparison:

- We use the restricted 2PN waveform; CF94 use the leading "Newtonian, quadrupole" waveform that we used for pedagogical purposes in Sec. 1.2. Since distance is encoded in the waveform's amplitude, we do not expect that our use of a higher-order phase function will have a large impact. However, to avoid any easily circumvented mismatch, we adopt the Newtonian-quadrupole waveform for these comparisons. This waveform does not depend on reduced mass $\mu$, so for the purpose of this comparison only, our parameter space is reduced from 7 to 6 dimensions.

- We use the projected advanced sensitivity noise curve shown in Fig. (1); CF94 use an analytical form [their Eq. (2.1) 11] based on the best-guess for what advanced sensitivity would achieve at the time of their analysis. Compared to the most recent projected sensitivity, their curve underestimates the noise at middle frequencies $(\sim 40-150$ $\mathrm{Hz})$ and overestimates it at high frequencies $(\gtrsim 200$ $\mathrm{Hz})$. We adopt their noise curve for this comparison. Because of these differences, CF94 rather seriously overestimates the SNR for NS-NS inspiral. Using their noise curve, the average SNR for the binary analyzed in their Fig. 10 is 12.412; using our up-to-date model for advanced LIGO, it is 5.8. As such, the reader should view the numbers in this section of our analysis as useful only for validation purposes.

- The two analyses use different priors. As extensively discussed in Sec. 3.3, we set uniform priors on the chirp mass $\mathcal{M}_{z}$, on the time $t_{c}$ and phase $\Phi_{c}$ at coalescence, and on the polarization phase $\psi$. For this comparison, we assume isotropic emission and set a flat prior on $\cos \iota$. We assume our sources are uniformly distributed in constant comoving volume. However, our detection threshold depends on the total network SNR, and effectively sets a joint prior on source inclination and distance. CF94 use a prior distribution only for the set $\left\{D_{L}, \cos \iota, \psi, \Phi_{c}\right\}$ that is flat in polarization phase, coalescence phase, and inclination. They assume a prior that is uniform in volume, but that cuts off the distribution at a distance $D_{L, \max } \simeq 6.5 \mathrm{Gpc}$.

Our goal here is to reproduce the 1-D marginalized posterior $\mathrm{PDF}$ in $D_{L}$ for the binary shown in Fig. 10 of CF94. We call this system the "CF binary." Each NS in the CF binary has $m_{z}=1.4 M_{\odot}$ and sky position $(\theta, \phi)=\left(50^{\circ}, 276^{\circ}\right)$; the detector network comprises LIGO Hanford, LIGO Livingston and Virgo. CF94 report the "best-fit" maximum-likelihood values $\left(\tilde{D}_{L}, \cos \tilde{\iota}\right.$, $\tilde{\Psi})$ to be $\left(432 \mathrm{Mpc}, 0.31,101.5^{\circ}\right)$, where $\Psi=\psi+\Delta \psi(\mathbf{n})$, and where $\Delta \psi(\mathbf{n})$ depends on the preferred basis of $\mathbf{e}^{\times}$ and $\mathbf{e}^{\times}$set by the detector network [see Eqs. (4.23)(4.25) of CF94 13 . To compare our distribution with theirs, we assume that $\hat{\boldsymbol{\theta}}=\tilde{\boldsymbol{\theta}}_{\mathrm{ML}}$ for the purpose of computing the likelihood function $\mathcal{L}(\boldsymbol{\theta} \mid \mathbf{s})$. This is a reasonable assumption when the priors are uniform over the relevant parameter space. As already mentioned, for this

11 Note that it is missing an overall factor of $1 / 5$ (E. E. Flanagan, private communication).

12 CF94 actually report an SNR of 12.8. The discrepancy is due to rounding the parameter $r_{0}$ in their Eq. (4.28). Adjusting to their preferred value (rather than computing $r_{0}$ ) gives perfect agreement.

13 Note that Eq. (4.25) of CF94 should read $\tan (4 \Delta \psi)=$ $2 \Theta_{+\times} /\left(\Theta_{++}-\Theta_{\times x}\right)$. In addition, $\tilde{\Psi}=56.5^{\circ}$ should read $\tilde{\Psi}=101.5^{\circ}$ under the caption of Fig. 10. (We have changed notation from $\bar{\psi}$ in CF94 to $\Psi$ to avoid multiple accents on the best fit value.) We thank Éanna Flanagan for confirming these corrections. 

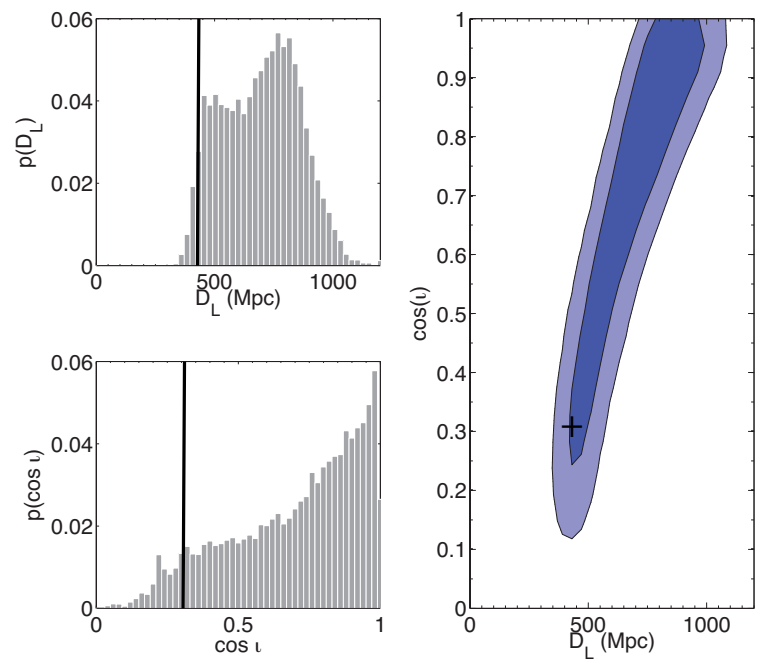

FIG. 5.- 1-D and 2-D marginalized posterior PDFs for $D_{L}$ and $\cos \iota$ averaged over noise (as described in Sec. 3.4) for the "CF binary." Our goal is to reproduce, as closely as possible, the nonGaussian limit summarized in Fig. 10 of CF94. Top left panel shows the 1-D marginalized posterior PDF in $D_{L}$ (the true value $\hat{D}_{L}=432 \mathrm{Mpc}$ is marked with a solid black line); bottom left panel illustrates the $1-\mathrm{D}$ marginalized posterior $\mathrm{PDF}$ in $\cos \iota$ (true value $\cos \hat{\iota}=0.31$ likewise marked). The right-hand panel shows the 2-D marginalized posterior PDF for $D_{L}$ and $\cos \iota$; the true values $\left(\hat{D}_{L}=432 \mathrm{Mpc}, \cos \hat{\imath}=0.31\right)$ are marked with a cross. The contours around the dark and light areas indicate the 68 and $95 \%$ interval levels, respectively. The true values lie within the $68 \%$ interval. The Bayes mean and rms measurement accuracies are (694.4 Mpc, 0.70) and (162 Mpc, 0.229) for $\left(D_{L}, \cos \iota\right)$, respectively.

comparison we use their advanced detector noise curve and the Newtonian-quadrupole waveform. Finally, we interpret the solid curve in Fig. 10 of CF94 as the marginalized 1-D posterior PDF in $D_{L}$ for an average of posterior PDFs of parameters (given an ensemble of many noisy observations for a particular event). We compute the average PDF as described in Sec. 3.4, and then marginalize over all parameters except $D_{L}$, using Eq. (60).

The left-hand panels of Fig. 5 show the resulting 1-D marginalized PDF in $D_{L}$ and $\cos \iota$. Its shape has a broad structure not dissimilar to the solid curve shown in Fig. 10 of CF94: The distribution has a small bump near $D_{L} \approx 460 \mathrm{Mpc}$, a main peak at $D_{L} \approx 700 \mathrm{Mpc}$, and extends out to roughly 1 Gigaparsec. Because of the broad shape, the Bayes mean $\left(\tilde{D}_{L, \text { BAYES }}=694 \mathrm{Mpc}\right)$ is significantly different from both the true value $\left(\hat{D}_{L}=432 \mathrm{Mpc}\right.$ in our calculation) and from the maximum likelihood $\left(\tilde{D}_{L, \mathrm{ML}}=495 \mathrm{Mpc}\right)$. Thanks to the marginalization, the peak of this curve does not coincide with the maximum likelihood.

We further determine the 2-D marginalized posterior PDFs in $D_{L}$ and $\cos \iota$ for the CF binary. Figure 5 illustrates directly the very strong degeneracy between these parameters, as expected from the form of Eqs. (7) and (8), as well as from earlier works (e.g., Marković 1993, CF94). It's worth noting that, as CF94 comment, this binary is measured particularly poorly. This is largely due to the fact that one polarization is measured far better than the other, so that the $D_{L^{-}}$- $\cos \iota$ degeneracy is essentially unbroken. This degeneracy is responsible for
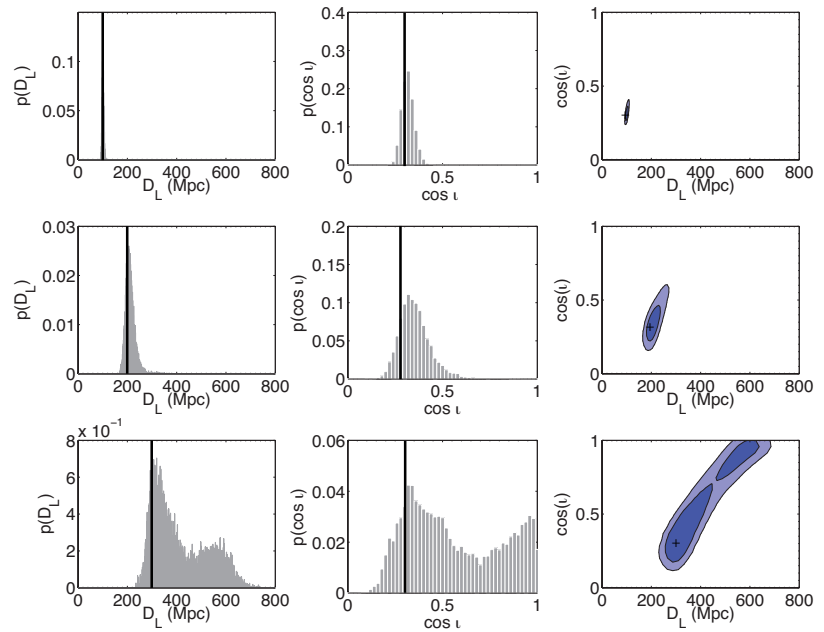

FIG. 6.- 1-D and 2-D marginalized PDFs for $D_{L}$ and $\cos \iota$, averaged (as described in Sec. 3.4) over noise ensembles for the "CF binary" at different values of true luminosity distance $\hat{D}_{L}$ : $[100$ $\mathrm{Mpc}, 200 \mathrm{Mpc}, 300 \mathrm{Mpc}$ ( top to bottom). True parameter values are marked with a solid black line or a black cross. The Bayes means and rms errors on luminosity distance are [101.0 Mpc, 212.1 $\mathrm{Mpc}, 411.2 \mathrm{Mpc}$ ] and [3.6 Mpc, 21.4 Mpc, 110.0 Mpc], respectively. The corresponding means and errors for $\cos \iota$ are $[0.317,0.357$, $0.562]$ and $[0.033,0.089,0.247]$. The dark and light contours in the 2-D marginalized PDF plots indicate the 68 and $95 \%$ interval levels, respectively. The true value always lies within the $68 \%$ contour region of the 2-D marginalized area at these distances.

the characteristic tail to large $D_{L}$ we find in the 1-D marginalized posterior PDF in $D_{L}, p\left(D_{L} \mid \mathbf{s}\right)$, which we investigate further in the following section.

\subsection{Test 1: Varying luminosity distance and number of detectors}

We now examine how well we measure $D_{L}$ as a function of distance to the CF binary and the properties of the GW detector network. Figures [6 and 7 show the 1$\mathrm{D}$ and 2-D marginalized posterior PDFs in $D_{L}$ and $\cos \iota$ for the CF binary at $\hat{D}_{L}=\{100,200,300,400,500$, $600\}$ Mpc. For all these cases, we keep the binary's sky position, inclination, and polarization angle fixed as in Sec. 4.1. The average network SNRs we find for these six cases are (going from $\hat{D}_{L}=100 \mathrm{Mpc}$ to $600 \mathrm{Mpc}$ ) 53.6, $26.8,17.9,13.4,10.7$, and 8.9 (scaling as $1 / \hat{D}_{L}$ ). Interestingly, the marginalized PDFs for both distance and $\cos \iota$ shown in Figs. 6] and 7 have fairly Gaussian shapes for $\hat{D}_{L}=100$ and $200 \mathrm{Mpc}$, but have very non-Gaussian shapes for $\hat{D}_{L} \geq 300 \mathrm{Mpc}$. This can be considered "anecdotal" evidence that the Gaussian approximation for the posterior PDF breaks down at SNR $\lesssim 25$ or so, at least for this case. For lower SNR, the degeneracy between $\cos \iota$ and $D_{L}$ becomes so severe that the 1-D errors on these parameters become quite large.

Next, we examine measurement accuracy versus detector network. For the CF binary, adding detectors does not substantially increase the total SNR. We increase the average total SNR from 12.4 to 14.6 (adding only AIGO), to 12.4 (adding only LCGT; its contribution is so small that the change is insignificant to the stated precision), or to 14.7 (adding both AIGO and LCGT). The average SNR in our detectors is 8.23 for LIGO-Hanford, 8.84 for 

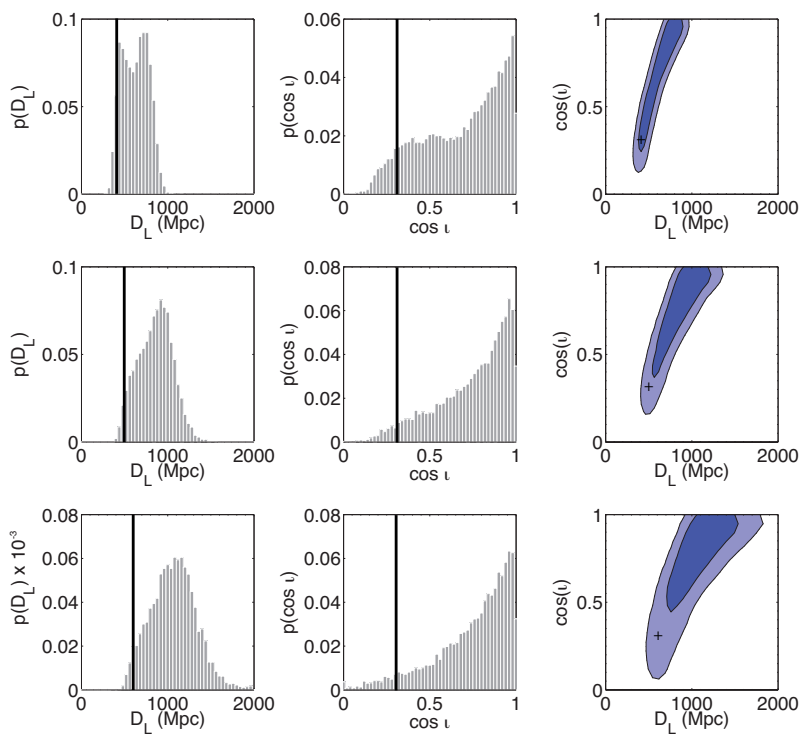

FIG. 7. - Same as Fig. 6] but for true luminosity distance $\hat{D}_{L}=$ [400 Mpc, $500 \mathrm{Mpc}, 600 \mathrm{Mpc}$ (top to bottom). True parameter values are marked with a solid black line or a black cross. In this case, the Bayes means and rms errors for luminosity distance are [627.17 Mpc, 857.3 Mpc, $1068 \mathrm{Mpc}$ ] and [148.8 Mpc, 198.1 Mpc, $262.2 \mathrm{Mpc}]$, respectively. The means and errors for $\cos \iota$ are $[0.686$, $0.745,0.746]$ and $[0.237,0.209,0.218]$. The dark and light contours in the 2-D marginalized PDF plots indicate the 68 and $95 \%$ interval levels, respectively. The true value lies within the $68 \%$ contour region for $D_{L}=400 \mathrm{Mpc}$, but moves outside this region for larger values.

LIGO-Livingston, 2.91 for Virgo, 8.71 for AIGO, and 1.1 for LCGT. This pathology is an example of a fairly general trend that we see; it is common for the SNR to be quite low in one or more detectors.

In the case of the CF binary, we find that adding detectors does not improve the measurement enough to break the $D_{L^{-}} \cos \iota$ degeneracy. The marginalized PDFs as functions of $D_{L}$ and $\cos \iota$ remain very similar to Fig. 5. so we do not show them. As a consequence, even with additional detectors, the distance errors remain large and biased. The bias is because we tend to find $\cos \iota$ to be larger than the true (relatively edge-on) value (cf. lower left-hand panel of Fig. 55). Thanks to the $D_{L}-\cos \iota$ degeneracy, we likewise overestimate distance.

\subsection{Test 2: Varying source inclination}

One of the primary results from the CF binary analysis is a strong degeneracy between $\cos \iota$ and $D_{L}$. As Fig. 5 shows, this results in a tail to large distance in the 1-D marginalized posterior PDF $p\left(D_{L} \mid \mathbf{s}\right)$, with a Bayes mean $\tilde{D}_{L}=694 \mathrm{Mpc}$ (compared to $\hat{D}_{L}=432 \mathrm{Mpc}$ ). Such a bias is of great concern for using binary sources as standard sirens.

The CF binary has $\cos \hat{\imath}=0.31$, meaning that it is nearly edge-on to the line of sight. Hypothesizing that the large tails may be due to its nearly edge-on nature, we consider a complementary binary that is nearly face on: We fix all of the parameters to those used for the CF binary, except for the inclination, which we take to be $\cos \hat{\imath}=0.98$. We call this test case the "face-on" CF binary. Changing to a more nearly face-on situation sub-
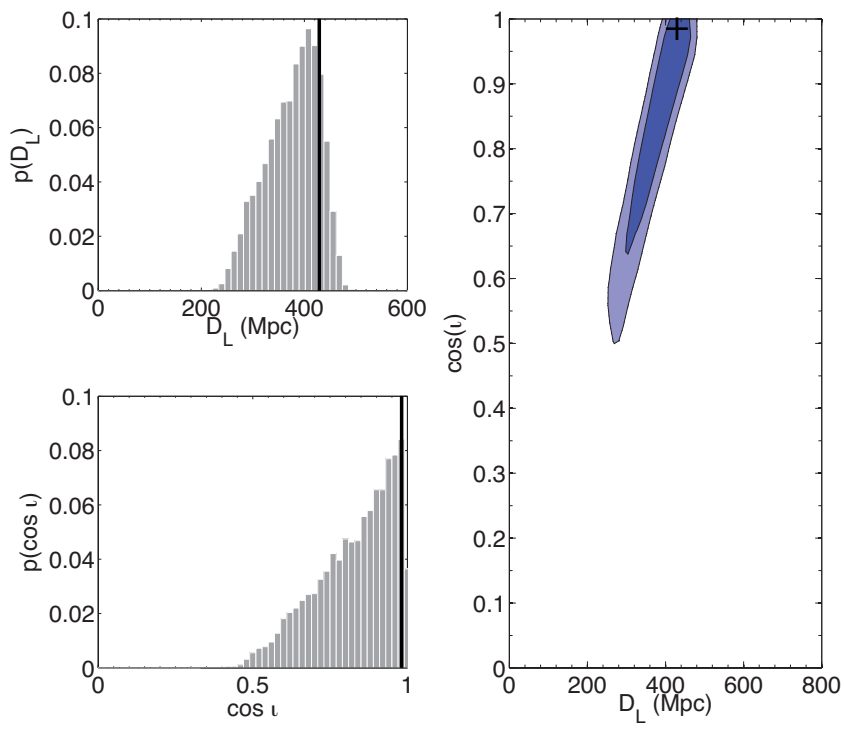

Fig. 8.- Same as Fig. [5 but for the "face-on" CF binary. The Bayes mean and rms errors are (376.3 Mpc, 0.83$)$ and $(51.3 \mathrm{Mpc}$, $0.12)$ for $\left(D_{L}, \cos \iota\right)$, respectively. Top left shows the 1-D marginalized posterior PDF in $D_{L}\left(\hat{D}_{L}=432 \mathrm{Mpc}\right.$ is marked with a solid black line); bottom left shows the marginalized PDF in $\cos \iota$ (solid black line marks $\cos \hat{\imath}=0.98$ ). The right panel shows the 2 -D marginalized posterior PDF; the cross marks the true source parameters $\left(\hat{D}_{L}=432 \mathrm{Mpc}, \cos \hat{\imath}=0.98\right)$. As with the CF binary, the true values lie within the $68 \%$ region.

stantially augments the measured SNR; the average SNR for the face-on CF binary measured by the LIGO/Virgo base network is 24.3 (versus 12.4 for the CF binary). We thus expect some improvement simply owing to the stronger signal.

Figure 8 shows the 1-D and 2-D marginalized posterior PDFs in $D_{L}$ and $\cos \iota$. As expected, these distributions are complementary to those we found for the CF binary. In particular, the peak of the 1-D marginalized posterior PDF in $D_{L}$ is shifted to lower values in $D_{L}$, and the Bayes mean is much closer to the true value: $\tilde{D}_{L}=376.3 \mathrm{Mpc}$. The shape of the 1-D marginalized posterior PDF in $\cos \iota$ is abruptly cut off by the upper bound of the physical prior $\cos \iota \leq 1$, and the tail extends to lower distances (the opposite of the CF binary). The Bayes mean for the inclination is $\cos \tilde{\iota}=0.83$.

Just as we varied distance and detector network for the CF binary, we also do so for the face-on CF binary, with very similar results. In particular, varying network has little impact on the marginalized 1-D PDFs in $D_{L}$ and $\cos \iota$. Varying distance, we find that the marginalized 1D PDFs are nearly Gaussian in shape for small distances, but become significantly skewed (similar to the left-hand panels of Fig. (8) when $\hat{D}_{L}>200 \mathrm{Mpc}$. The distributions in $\cos \iota$ are particularly skewed thanks to the hard cut-off at $\cos \iota=1$. Interestingly, in this case we tend to infer a value of $\cos \iota$ that is smaller than the true value. We likewise find a Bayes mean $\tilde{D}_{L}$ that is smaller than $\hat{D}_{L}$.

\subsection{Summary of validation tests}

The main result from our testing is that the posterior PDFs we find have rather long tails, with strong correlations between $\cos \iota$ and $D_{L}$. Except for cases with 
very high SNR, the 1-D marginalized posterior PDF in $\cos \iota$ tends to be rather broad. The Bayes mean for $\cos \iota$ thus typically suggests that a binary is at intermediate inclination. As such, we tend to underestimate $\cos \iota$ for nearly face-on binaries, and to overestimate it for nearly edge-on binaries. Overcoming this limitation requires us to either break the $D_{L}-\cos \iota$ degeneracy (such as by setting a prior on binary inclination), or by measuring a population of coalescences. Measuring a population will make it possible to sample a wide range of the $\cos \iota$ distribution, so that the event-by-event bias is averaged away in the sample.

\section{RESULTS II: SURVEY OF STANDARD SIRENS}

We now examine how well various detector networks can measure an ensemble of canonical GW-SHB events. We randomly choose events from our sample of detected NS-NS and NS-BH binaries (where the selection is detailed in Sec. 3.2). We set a total detector network threshold of 7.5. Crudely speaking, one might imagine that this implies, on average, a threshold per detector of $7.5 / \sqrt{5}=3.4$ for a five detector network. Such a crude "per detector threshold" is useful for getting a rough idea of the range to which our network can measure events. Averaging Eq. (45) over all sky positions and orientations yields (DHHJ06)

$$
\begin{gathered}
\left(\frac{S}{N}\right)_{a, \text { sky }- \text { ave }}=\frac{8}{5} \sqrt{\frac{5}{96}} \frac{c}{D_{L}} \frac{1}{\pi^{2 / 3}}\left(\frac{G \mathcal{M}_{z}}{c^{3}}\right)^{5 / 6} \times \\
\int_{f_{\text {low }}}^{f_{\text {ISCO }}} \frac{f^{-7 / 3}}{S_{h}(f)} d f,
\end{gathered}
$$

For total detector network threshold of 7.5, a five detector network has an average range of about $600 \mathrm{Mpc}$ for NS-NS events, and about $1200 \mathrm{Mpc}$ for NS-BH events. If SHBs are associated with face-on binary inspiral, these numbers are increased by a factor $\sqrt{5 / 2} \simeq 1.58$. (This factor is incorrectly stated to be $\sqrt{5 / 4} \simeq 1.12$ in DHHJ06.)

Let us assume a constant comoving rate of 10 SHBs $\mathrm{Gpc}^{3} \mathrm{yr}^{-1}$ (Nakar et al. 2006). If these events are all NS-NS binary mergers, and they are isotropically oriented, we expect the full LIGO-Virgo-AIGO-LCGT network to measure $6 \mathrm{GW}-\mathrm{SHB}$ events per year. If these events are instead all NS-BH binaries, the full network is expected to measure 44 events per year. If these events are beamed, the factor 1.58 increases the expected rate to $9 \mathrm{NS}-\mathrm{NS}$ or $70 \mathrm{NS}-\mathrm{BH}$ GW-SHB events per year. We stress that these numbers should be taken as rough indicators of what the network may be able to measure. Not all SHBs will be associated with binary inspiral. Those events which are will likely include both NS-NS and NS$\mathrm{BH}$ events, with parameters differing from our canonical choices. We also do not account for the fraction of SHBs which will be missed due to incomplete sky coverage.

In all cases we build our results by constructing the posterior distribution for an event given a unique noise realization at each detector. We keep the noise realization in a given detector and for a specific binary constant as we add other detectors. This allows us to make meaningful comparisons between the performance of different detector networks.

\subsection{NS-NS binaries}

We begin by imagining a population of six hundred detected NS-NS binaries, either isotropically distributed in inclination angle or from our beamed subsample, using a network with all five detectors. Figure 9 shows scatter plots of the distance measurement accuracies for our unbeamed (blue crosses) and beamed events (black dots), with each panel corresponding to a different detector network. The distance measurement error is defined as the ratio of the rms measurement error with the true value ${ }^{14}$ $\hat{D}_{L}$ :

$$
\frac{\Delta D_{L}}{\hat{D}_{L}}=\frac{\sqrt{\Sigma^{D_{L} D_{L}}}}{\hat{D}_{L}} .
$$

$\Sigma^{D_{L} D_{L}}$ is computed using (51). We emphasize some general trends in Fig. 9 which are particularly relevant to standard sirens:

- The unbeamed total sample and the beamed subsample separate into two distinct distributions. As anticipated, the beamed subsample improves measurement errors in $D_{L}$ significantly, by greater than a factor of two or more. This is due to the beaming prior, which constrains the inclination angle, $\cos \iota$, to $\sim 3 \%$, thereby breaking the strong $D_{L}-\cos \iota$ degeneracy. By contrast, when no beaming prior is assumed, we find absolute errors of $0.1-0.3$ in $\cos \iota$ for the majority of events. The strong $D_{L}-\cos \iota$ degeneracy then increases the distance errors. A significant fraction of binaries randomly selected from our sample have $0.5 \lesssim|\cos \hat{\imath}|<1$. As discussed in Sec. 3.2, this is due to the SNR selection criterion: At fixed distance, face-on binaries are louder and tend to be preferred.

- Beamed subsample scalings. We fit linear scalings to our beamed subsample:

$\Delta D_{L} / \hat{D}_{L} \simeq \hat{D}_{L} /(2.15 \mathrm{Gpc})$ for LIGO + Virgo

$\Delta D_{L} / \hat{D}_{L} \simeq \hat{D}_{L} /(2.71 \mathrm{Gpc})$ for $\mathrm{LIGO}+\mathrm{Virgo}+$ AIGO

$\Delta D_{L} / \hat{D}_{L} \simeq \hat{D}_{L} /(2.38 \mathrm{Gpc})$ for $\mathrm{LIGO}+$ Virgo + LCGT

$\Delta D_{L} / \hat{D}_{L} \simeq \hat{D}_{L} /(2.82 \mathrm{Gpc})$ for $\mathrm{LIGO}+$ Virgo + $\mathrm{AIGO}+\mathrm{LCGT}$

- When isotropic emission is assumed, we find a large scatter in distance measurement errors for all events, irrespective of network and true distance. We find much less scatter when we assume a beaming prior. This is illustrated very clearly by the upper-right panel of Fig. 9. In that panel, we show the scatter of distance measurement error versus true distance for the LIGO, Virgo, AIGO detector network, comparing to the Fisher-matrix-derived linear scaling trend found in DHHJ06. For the unbeamed case, our current results scatter around the linear trend; for the beamed case, most events lie fairly close to the trend. This demonstrates starkly

14 Our definition differs from that given in CF94, their Eq. (4.62). Their distance measurement error is described as the ratio of the rms measurement error with the Bayes mean. We prefer to use Eq. (62) as we are interested primarily in the measurement error given a binary at its true luminosity distance. 

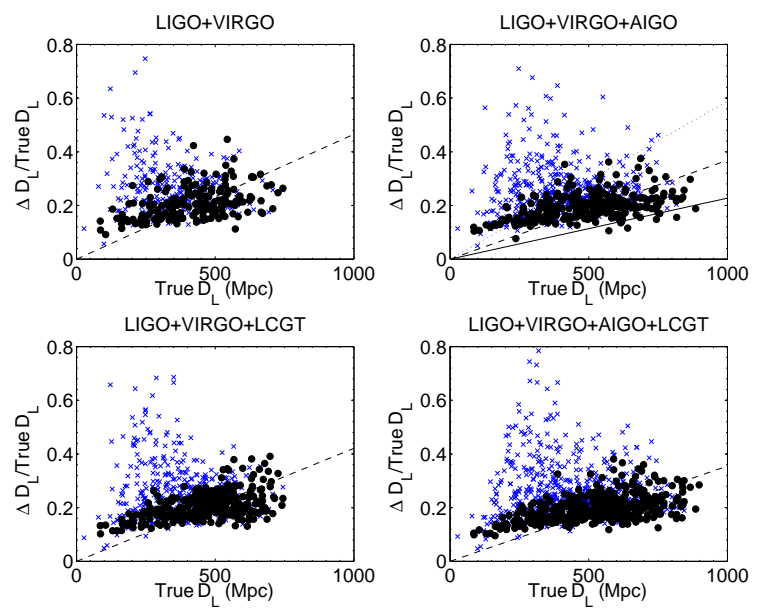

FIG. 9.- Distance measurement errors versus true luminosity distance for our sample of NS-NS binaries. Colored crosses assume isotropic emission; black points assume our beaming prior. The dashed lines show the linear best-fit to the beamed sample (see text for expressions). In the LIGO+Virgo+AIGO panel we also show the Fisher-matrix-derived linear scaling given in DHHJ06: $\Delta D_{L} / \hat{D}_{L} \simeq \hat{D}_{L} /(4.4 \mathrm{Gpc})$ assuming beaming (solid), and $\Delta D_{L} / \hat{D}_{L} \simeq \hat{D}_{L} /(1.7 \mathrm{Gpc})$ for isotropic emission (dotted).

the failure of Fisher methods to estimate distance accuracy, especially when we cannot set a beaming prior.

- Adding detectors to the network considerably increases the number of detected binaries, but does not significantly improve the accuracy with which those binaries are measured. The increase we see in the number of detected binaries is particularly significant for GW-SHB standard sirens. For instance, an important application is mapping out the posterior PDF for the Hubble constant, $H_{0}$. As the number of events increases, the resulting joint posterior PDF in $H_{0}$ will become increasingly well constrained. Additional detectors also increase the distance to which binaries can be detected. This can be seen in Fig. 9. for the LIGO and Virgo network, our detected events extend to $\hat{D}_{L} \sim 600 \mathrm{Mpc}$; the larger networks all go somewhat beyond this. Interestingly, networks which include the AIGO detector seem to reach somewhat farther out.

It is perhaps disappointing that increasing the number of detectors does not greatly improve measurement accuracy. We believe this is due to two effects. First, a larger network tends to detect more weak signals. These additional binaries are poorly constrained. Second, the principle limitation to distance measurement is the $D_{L^{-}}$ $\cos \iota$ degeneracy. A substantial improvement in distance accuracy on individual events would require breaking this degeneracy. We find that adding detectors does not do this, but the beaming prior does.

\section{2. $N S-B H$ binaries}

We now repeat the preceding analysis for six hundred detected NS-BH binaries. Figure 10 shows scatter plots of measurement accuracies for unbeamed and beamed NS-BH binaries. We find similar trends to the NS-NS case:
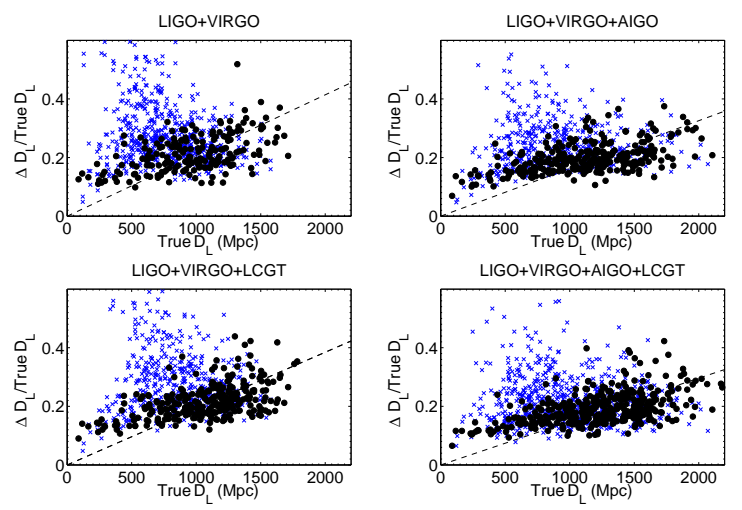

FIG. 10.- Distance measurement errors versus true luminosity distance for our sample of NS-BH binaries. Colored crosses assume isotropic emission; black points use our beaming prior. The lower right-hand panel shows the sample detected by our "full" network (LIGO+Virgo+AIGO+LCGT). Upper left is LIGO+Virgo; upper right is LIGO+Virgo+AIGO; and lower left is LIGO+Virgo+LCGT. The dashed lines show the linear best-fit to the beamed sample (see text for expressions).

- The unbeamed and beamed samples separate into two distinct distributions. Notice, however, that outliers exist in measurement errors at high $D_{L}$ for several beamed events for all networks. This is not too surprising, given that we expect beamed sources at higher luminosity distances and lower SNR. Such events are more likely to deviate from the linear relationship predicted by the Fisher matrix.

- We see substantial scatter in distance measurement, particularly when isotropic emission is assumed. As with the NS-NS case, the scatter is not as severe when we assume beaming, and in that case lies fairly close to a linear trend, as would be predicted by a Fisher matrix. This trend is shallower in slope than for NS-NS binaries, thanks to the larger mass of the system.

- We do not see substantial improvement in distance measurement as we increase the detector network. As with NS-NS binaries, adding detectors increases the range of the network; AIGO appears to particularly add events at large $\hat{D}_{L}$ (for both the isotropic and beamed samples). However, adding detectors does not break the fundamental $D_{L}-\cos \iota$ degeneracy, and doesn't improve errors. From our full posterior PDFs, we find absolute errors of $0.1-0.3$ in $\cos \iota$, which is very similar to the NS-NS case.

- Beamed subsample scalings. The linear scalings for our beamed subsample are:

$\Delta D_{L} / \hat{D}_{L} \simeq \hat{D}_{L} /(4.83 \mathrm{Gpc})$ for LIGO + Virgo

$\Delta D_{L} / \hat{D}_{L} \simeq \hat{D}_{L} /(6.14 \mathrm{Gpc})$ for LIGO + Virgo + AIGO

$\Delta D_{L} / \hat{D}_{L} \simeq \hat{D}_{L} /(5.20 \mathrm{Gpc})$ for LIGO + Virgo + LCGT

$\Delta D_{L} / \hat{D}_{L} \simeq \hat{D}_{L} /(6.76 \mathrm{Gpc})$ for $\mathrm{LIGO}+\mathrm{Virgo}+$ $\mathrm{AIGO}+\mathrm{LCGT}$ 


\section{SUMMARY DISCUSSION}

In this analysis we have studied how well GWs can be used to measure luminosity distance, under the assumption that binary inspiral is associated with (at least some) short-hard gamma ray bursts. We examine two plausible compact binary SHB progenitors, and a variety of plausible detector networks. We emphasize that we assume sky position is known. We build on the previous study of DHHJ06, which used the so-called Gaussian approximation of the posterior PDF. This approximation works well for large SNR, but the limits of its validity are poorly understood. In particular, since the SNR of events measured by ground-based detectors is likely to be of order 10, the Gaussian limit may be inapplicable. We examine the posterior PDF for the parameters of observed events using Markov-Chain Monte-Carlo techniques, which do not rely on this approximation. We also introduce a welldefined noise-averaged posterior PDF that does not depend solely on a particular noise instance. Such a quantity is useful to predict how well a detector should be able to measure the properties of a source.

We find that the Gaussian approximation substantially underestimates distance measurement errors. We also find that the main limitation for individual standard siren measurements is the strong degeneracy between distance to the binary and the binary's inclination to the line of sight; similar discussion of this issue is given in a recent analysis by Ajith \& Bose (2009). Adding detectors to a network only slightly improves distance measurement for a given single event. When we assume that the SHB is isotropic (so that we cannot infer anything about the source's inclination from the burst), we find that Fisher matrix estimates of distance errors are very inaccurate. Our distributions show large scatter about the Fisher-based predictions.

The situation improves dramatically if we assume that SHBs are collimated, thereby giving us a prior on the orientation of the progenitor binary. By assuming that SHBs are preferentially emitted into an opening angle of roughly $25^{\circ}$, we find that the distance-inclination correlation is substantially broken. The Fisher matrix estimates are then much more reasonable, giving a good sense of the trend with which distances are determined (albeit with a moderate scatter about that trend). This illustrates the importance of incorporating prior knowledge, at least for individual measurements.

Our distance measurement results are summarized by Fig. 9 (for NS-NS SHB progenitors) and Fig. 10 (for NS$\mathrm{BH})$. Assuming isotropy, we find the distance to NS-NS binaries is measured with a fractional error of roughly 20-60\%, with most events in our distribution clustered near $20-30 \%$. Beaming improves this by roughly a factor of two, and eliminates much of the high error tail from our sample. NS-BH events are measured somewhat more accurately: the distribution of fractional distance errors runs from roughly $15-50 \%$, with most events clustered near $15-25 \%$. Beaming again gives roughly a factor of two improvement, elimating most of the high error tail.

It is worth emphasizing that these results describe the outcome of individual siren measurements. When these measurements are used as cosmological probes, we will be interested in constructing the joint distribution, following observation of $N \mathrm{GW}$-SHB events. Indeed, preliminary studies show that our ability to constrain $H_{0}$ improves dramatically as the number of measured binaries is increased. In our most pessimistic scenario (the SHB is assumed to be a NS-NS binary, with no prior on inclination, and measured by the baseline LIGO-Virgo network), we find that $H_{0}$ can be measured with $\sim 13 \%$ fractional error with $N=4$, improving to $\sim 5 \%$ for $N=15$. This is because multiple measurements allow us to sample the inclination distribution, and thus average out the bias introduced by the tendency to overestimate distance for edge-on binaries, and underestimate it for face-on binaries. Details of this analysis will be presented in a followup paper.

Increasing the number of measured events will thus be crucial for making cosmologically interesting measurements. To this end, it is important to note that increasing the number of detectors in our network enables a considerable increase in the number of detected binaries. This is due to increases in both the sky coverage and in the total detection volume. Going from a network which includes all four detectors (LIGO, Virgo, AIGO, and LCGT) to our baseline network of just LIGO and Virgo entails a $\sim 50 \%$ reduction in the number of detected binaries. Eliminating just one of the proposed detectors (AIGO or LCGT) leaves us with $\sim 75 \%$ of the original detected sample.

Aside from exploring the cosmological consequences, several other issues merit careful future analysis. One general result is the importance that priors have on the posterior PDF. We plan to examine this in some detail, identifying the parameters which particularly influence the final result, and which uncertainties can be ascribed to an inability to set relevant priors. Another issue is the importance of systematic errors in these models. We have used the second-post-Newtonian description of a binary's GWs in this analysis, and have ignored all but the leading quadrupole harmonic of the waves (the "restricted" post-Newtonian waveform). Our suspicion is that a more complete post-Newtonian description of the phase would have little impact on our results, since such effects won't impact the $D_{L}-\cos \iota$ degeneracy. In principle, including additional (non-quadrupole) harmonics could have an impact, since these other harmonics encode different information about the inclination angle $\iota$. In practice, we expect that they won't have much effect on GW-SHB measurements, since these harmonics are measured with very low SNR (the next strongest harmonic is roughly a factor of 10 smaller in amplitude than the quadrupole).

As discussed previously, we confine our analysis to the inspiral. Inspiral waves are terminated at the innermost stable circular orbit frequency, $f_{\mathrm{ISCO}}=\left(6^{3 / 2} \pi M_{z}\right)$. For NS-NS binaries, $f_{\text {ISCO }} \simeq 1600 \mathrm{~Hz}$. At this frequency, detectors have fairly poor sensitivity, so we are confident that terminating the waves has little impact on our NSNS results. However, for our assumed NS-BH binaries, $f_{\text {ISCO }} \simeq 400 \mathrm{~Hz}$. Detectors have good sensitivity in this band, so it may be quite important to improve our model for the waves' termination in this case.

Perhaps the most important follow-up would be to include the impact of spin. Although the impact of neutron star spin is likely to be small, it may not be negligible; and, for NS-BH systems, the impact of the black 
hole's spin is likely to be significant. Spin induces precession which makes the orbit's orientation, $\hat{\mathbf{L}}$, dynamical. That makes the observed inclination dynamical, which can break the $D_{L}-\cos \iota$ degeneracy. In other words, with spin precession the source's orbital dynamics may break this degeneracy. Van der Sluys et al. (2008) have already shown that spin precession physics can improve the ability of ground-based detectors to determine a source's position on the sky. We are confident that a similar analysis which assumes known sky position will find that measurements of source distance and inclination can likewise be improved.

It is a pleasure to acknowledge useful discussions with K. G. Arun, Yoicho Aso, Duncan Brown, Curt Cutler, Jean-Michel Désert, Alexander Dietz, L. Samuel Finn, Derek Fox, Éanna Flanagan, Zhiqi Huang, Ryan Lang, Antony Lewis, Ilya Mandel, Nergis Mavalvala, Szabolcs
Márka, Phil Marshall, Cole Miller, Peng Oh, Ed Porter, Alexander Shirokov, David Shoemaker, and Pascal Vaudrevange. We are grateful to Neil Cornish in particular for early guidance on the development of our MCMC code, to Michele Vallisneri for careful reading of the manuscript, and to Phil Marshall for his detailed comments on the ensemble averaged likelihood function. We also are grateful for the hospitality of the Kavli Institute for Theoretical Physics at UC Santa Barbara, and to the Aspen Center for Physics, where portions of the work described here were formulated. Computations were performed using the Sunnyvale computing cluster at the Canadian Institute for Theoretical Astrophysics, which is funded by the Canadian Foundation for Innovation. SAH is supported by NSF Grant PHY-0449884, and the MIT Class of 1956 Career Development Fund. He gratefully acknowledges the support of the Adam J. Burgasser Chair in Astrophysics.

\section{REFERENCES}

Abadie, J., Abbott, B. P., Abbott, R., Abernathy, M., Accadia, T., Acernese, F., Adams, C., Adhikari, R., Ajith, P., Allen, B., \& et al. 2010, Classical and Quantum Gravity, 27, 173001

Acernese, F., \& Virgo Scientific Collaboration. 2008, Classical and Quantum Gravity, 25, 184001

Ajith, P., \& Bose, S. 2009, Phys. Rev. D, 79, 084032

Althouse, W., Jones, L., \& Lazzerini, A. 1998, Technical Report No. LIGO-T980044-08

Anderson, W. G., Brady, P. R., Creighton, J. D., \& Flanagan, É. É. 2001, Phys. Rev. D, 63, 042003

Barriga, P., Blair, D. G., Coward, D., Davidson, J., Dumas, J., Howell, E., Ju, L., Wen, L., Zhao, C., McClelland, D. E., Scott, S. M., Slagmolen, B. J. J., Inta, R., Munch, J., Ottaway, D. J., Veitch, P., Hosken, D., Melatos, A., Chung, C., Sammut, L., Galloway, D. K., Marx, J., Whitcomb, S., Shoemaker, D., Hughes, S. A., Reitze, D. H., Iyer, B. R., Dhurandhar, S. V., Souradeep, T., Unnikrishnan, C. S., Rajalakshmi, G., Man, C. N., Heidmann, A., Cohadon, P., Briant, T., Grote, H., Danzmann, K., Lück, H., Willke, B., Strain, K. A., Sathyaprakash, B. S., Cao, J., Cheung, Y., \& Zhang, Y. 2010, Classical and Quantum Gravity, 27, 084005

Berger, E., Fox, D. B., Price, P. A., Nakar, E., Gal-Yam, A., Holz, D. E., Schmidt, B. P., Cucchiara, A., Cenko, S. B., Kulkarni, S. R., Soderberg, A. M., Frail, D. A., Penprase, B. E., Rau, A., Ofek, E., Burnell, S. J. B., Cameron, P. B., Cowie, L. L., Dopita, M. A., Hook, I., Peterson, B. A., Podsiadlowski, P., Roth, K. C., Rutledge, R. E., Sheppard, S. S., \& Songaila, A. 2007, ApJ, 664, 1000

Blair, D. G., Barriga, P., Brooks, A. F., Charlton, P., Coward, D., Dumas, J.-C., Fan, Y., Galloway, D., Gras, S., Hosken, D. J., Howell, E., Hughes, S., Ju, L., McClelland, D. E., Melatos, A., Miao, H., Munch, J., Scott, S. M., Slagmolen, B. J. J., Veitch, P. J., Wen, L., Webb, J. K., Wolley, A., Yan, Z., \& Zhao, C. 2008, Journal of Physics Conference Series, 122, 012001

Blanchet, L. 2006, Living Reviews in Relativity, 9, 4

Blanchet, L., Damour, T., Esposito-Farèse, G., \& Iyer, B. R. 2004, Physical Review Letters, 93, 091101

Blanchet, L., Damour, T., Iyer, B. R., Will, C. M., \& Wiseman, A. G. 1995, Phys. Rev. Lett., 74, 3515

Blanchet, L., Faye, G., Iyer, B. R., \& Joguet, B. 2002a, Phys. Rev. D, 65, 061501

Blanchet, L., Iyer, B. R., \& Joguet, B. 2002b, Phys. Rev. D, 65, 064005
Bloom, J. S., Holz, D. E., Hughes, S. A., Menou, K., Adams, A., Anderson, S. F., Becker, A., Bower, G. C., Brandt, N., Cobb, B., Cook, K., Corsi, A., Covino, S., Fox, D., Fruchter, A., Fryer, C., Grindlay, J., Hartmann, D., Haiman, Z., Kocsis, B., Jones, L., Loeb, A., Marka, S., Metzger, B., Nakar, E., Nissanke, S., Perley, D. A., Piran, T., Poznanski, D., Prince, T., Schnittman, J., Soderberg, A., Strauss, M., Shawhan, P. S., Shoemaker, D. H., Sievers, J., Stubbs, C., Tagliaferri, G., Ubertini, P., \& Wozniak, P. 2009, ArXiv e-prints

Burrows, D. N., Grupe, D., Capalbi, M., Panaitescu, A., Patel, S. K., Kouveliotou, C., Zhang, B., Mészáros, P., Chincarini, G., Gehrels, N., \& Wijers, R. A. M. 2006, ApJ, 653, 468

Cavalier, F., Barsuglia, M., Bizouard, M.-A., Brisson, V., Clapson, A.-C., Davier, M., Hello, P., Kreckelbergh, S., Leroy, N., \& Varvella, M. 2006, Phys. Rev. D, 74, 082004

Christensen, N., Meyer, R., \& Libson, A. 2004, Class. Quantum Grav., 21, 317

Cook, G. B., Shapiro, S. L., \& Teukolsky, S. A. 1994, ApJ, 424, 823

Cornish, N. J., \& Porter, E. K. 2007, Classical and Quantum Gravity, 24, 5729

Cutler, C. 1998, Phys. Rev. D, 57, 7089

Cutler, C., \& Flanagan, É. E. 1994, Phys. Rev. D, 49, 2658, referred to in the text as CF94

Cutler, C., \& Vallisneri, M. 2007, Phys. Rev. D, 76, 104018

Dalal, N., Holz, D. E., Hughes, S. A., \& Jain, B. 2006, Phys. Rev. D, 74, 063006, referred to in the text as DHHJ06 Dietz, A. 2009, ArXiv e-prints

Droz, S., Knapp, D. J., Poisson, E., \& Owen, B. J. 1999,

Phys. Rev. D, 59, 124016

Dunkley, J., Komatsu, E., Nolta, M. R., Spergel, D. N., Larson, D., Hinshaw, G., Page, L., Bennett, C. L., Gold, B., Jarosik, N., Weiland, J. L., Halpern, M., Hill, R. S., Kogut, A., Limon, M., Meyer, S. S., Tucker, G. S., Wollack, E., \& Wright, E. L. 2009, ApJS, 180, 306

Eichler, D., Livio, M., Piran, T., \& Schramm, D. N. 1989, Nature, 340,126

Etienne, Z. B., Faber, J. A., Liu, Y. T., Shapiro, S. L., Taniguchi, K., \& Baumgarte, T. W. 2008, Phys. Rev. D, 77, 084002

Fairhurst, S. 2009, New Journal of Physics, 11, 123006

Finn, L. S. 1992, Phys. Rev. D, 46, 5236

Finn, L. S., \& Chernoff, D. F. 1993, Phys. Rev. D, 47, 2198

Ford, E. B. 2005, AJ, 129, 1706 
Fox, D. B., Frail, D. A., Price, P. A., Kulkarni, S. R., Berger, E., Piran, T., Soderberg, A. M., Cenko, S. B., Cameron, P. B., Gal-Yam, A., Kasliwal, M. M., Moon, D.-S., Harrison, F. A., Nakar, E., Schmidt, B. P., Penprase, B., Chevalier, R. A., Kumar, P., Roth, K., Watson, D., Lee, B. L., Shectman, S., Phillips, M. M., Roth, M., McCarthy, P. J., Rauch, M., Cowie, L., Peterson, B. A., Rich, J., Kawai, N., Aoki, K., Kosugi, G., Totani, T., Park, H.-S., MacFadyen, A., \& Hurley, K. C. 2005, Nature, 437, 845

Gelman, A., \& Rubin, D. B. 1992, Statistical Science, 4, 457

Gilks, W. R., Richardson, S., \& Spiegelhalter, D. J. 1996, Markov Chain Monte Carlo in Practice (Markov Chain Monte Carlo in Practice, by W. R. Gilks, S. Richardson and

D. J. Spiegelhalter, pp. 486. ISBN 0412055511. London, UK: Chapman and Hall, 1996.)

Grupe, D., Burrows, D. N., Patel, S. K., Kouveliotou, C., Zhang, B., Mészáros, P., Wijers, R. A. M., \& Gehrels, N. 2006, ApJ, 653,462

Harry, G. M., \& LIGO Scientific Collaboration. 2010, Classical and Quantum Gravity, 27, 084006

Hastings, W. K. 1970, Biometrika, 57, 97

Hogg, D. W. 1999, ArXiv Astrophysics e-prints

Holz, D. E., \& Hughes, S. A. 2005, ApJ, 629, 15

Kochanek, C. S., \& Piran, T. 1993, ApJ, 417, L17

Komatsu, E., Dunkley, J., Nolta, M. R., Bennett, C. L., Gold, B., Hinshaw, G., Jarosik, N., Larson, D., Limon, M., Page, L., Spergel, D. N., Halpern, M., Hill, R. S., Kogut, A., Meyer, S. S., Tucker, G. S., Weiland, J. L., Wollack, E., \& Wright, E. L. 2009, ApJS, 180, 330

Kopparapu, R. K., Hanna, C., Kalogera, V., O’Shaughnessy, R., González, G., Brady, P. R., \& Fairhurst, S. 2008, ApJ, 675, 1459

Królak, A., Lobo, J. A., \& Meers, B. J. 1993, Phys. Rev. D, 48, 3451

Kulkarni, S., \& Kasliwal, M. M. 2009, ArXiv e-prints

Kuroda, K., \& LCGT Collaboration. 2010, Classical and Quantum Gravity, 27, 084004

Lang, R. N., \& Hughes, S. A. 2006, Phys. Rev. D, 74, 122001

Lewis, A., \& Bridle, S. 2002, Phys. Rev. D, 66, 103511

Loredo, T. J. 1989, in Bulletin of the American Astronomical Society, Vol. 21, Bulletin of the American Astronomical Society, 1136

MacKay, D. J. C. 2003, Information Theory, Inference and Learning Algorithms (Information Theory, Inference and Learning Algorithms, by David J. C. MacKay, pp. 640. ISBN 0521642981. Cambridge, UK: Cambridge University Press, October 2003.)

Marković, D. 1993, Phys. Rev. D, 48, 4738

Metropolis, N., Rosenbluth, A. W., Rosenbluth, M. N., Teller, A. H., \& Teller, E. 1953, J. Chem. Phys., 21, 1087

Nakar, E., Gal-Yam, A., \& Fox, D. B. 2006, Astrophys. J., 650, 281

Neal, R. M. 1993, Technical Report CRG-TR-93-1

Owen, B. J. 1996, Phys. Rev. D, 53, 6749
Peebles, P. J. E. 1993, Principles of physical cosmology (Princeton Series in Physics, Princeton, NJ: Princeton University Press, - c1993)

Perley, D. A., Metzger, B. D., Granot, J., Butler, N. R. Sakamoto, T., Ramirez-Ruiz, E., Levan, A. J., Bloom, J. S., Miller, A. A., Bunker, A., Chen, H.-W., Filippenko, A. V., Gehrels, N., Glazebrook, K., Hall, P., Hurley, K. C., Kocevski, D., Li, W., Lopez, S., Norris, J., Piro, A. L., Poznanski, D., Prochaska, J. X., Quataert, E., \& Tanvir, N. 2008, ArXiv e-prints

Phinney, E. S. 2009, ArXiv e-prints

Poisson, E., \& Will, C. M. 1995, Phys. Rev. D, 52, 848

Porter, E. K., \& Cornish, N. J. 2008, Phys. Rev. D, 78, 064005

Pretorius, F. 2005, Phys. Rev. Lett., 95, 121101

Raymond, V., van der Sluys, M. V., Mandel, I., Kalogera, V., Röver, C., \& Christensen, N. 2009, Classical and Quantum Gravity, 26, 114007

Röver, C., Meyer, R., \& Christensen, N. 2007, Phys. Rev. D, 75, 062004

Sathyaprakash, B. S., Schutz, B., \& Van Den Broeck, C. 2009, ArXiv e-prints

Schutz, B. F. 1986, Nature, 323, 310

Shibata, M., \& Uryū, K. 2006, Phys. Rev. D, 74, 121503

Sievers, J. L., Mason, B. S., Weintraub, L., Achermann, C., Altamirano, P., Bond, J. R., Bronfman, L., Bustos, R., Contaldi, C., Dickinson, C., Jones, M. E., May, J., Myers, S. T., Oyarce, N., Padin, S., Pearson, T. J., Pospieszalski, M., Readhead, A. C. S., Reeves, R., Shepherd, M. C., Taylor, A. C., \& Torres, S. 2009, ArXiv e-prints

Soderberg, A. M., Berger, E., Kasliwal, M., Frail, D. A., Price, P. A., Schmidt, B. P., Kulkarni, S. R., Fox, D. B., Cenko, S. B., Gal-Yam, A., Nakar, E., \& Roth, K. C. 2006, ApJ, 650, 261

Stroeer, A., Gair, J., \& Vecchio, A. 2006, in American Institute of Physics Conference Series, Vol. 873, Laser Interferometer Space Antenna: 6th International LISA Symposium, ed. S. M. Merkovitz \& J. C. Livas, 444-451

Sylvestre, J. 2004, Class. Quantum Grav., 21, 775

Thorne, K. S. 1987, in 300 Years of Gravitation, ed. S. W. Hawking \& W. Israel (Cambridge: Cambridge University Press), 330-458

Vallisneri, M. 2008, Phys. Rev. D, 77, 042001

van der Sluys, M., Mandel, I., Raymond, V., Kalogera, V., Röver, C., \& Christensen, N. 2009, Classical and Quantum Gravity, 26,204010

van der Sluys, M. V., Röver, C., Stroeer, A., Raymond, V., Mandel, I., Christensen, N., Kalogera, V., Meyer, R., \& Vecchio, A. 2008, ApJ, 688, L61

Vecchio, A. 2004, Phys. Rev. D, 70, 042001

Wen, L., \& Chen, Y. 2010, Phys. Rev. D, 81, 082001

Wickham, E. D. L., Stroeer, A., \& Vecchio, A. 2006, Classical and Quantum Gravity, 23, 819

Winn, J. N., Holman, M. J., Bakos, G. Á., Pál, A., Johnson, J. A., Williams, P. K. G., Shporer, A., Mazeh, T., Fernandez, J., Latham, D. W., \& Gillon, M. 2007, AJ, 134, 1707 